Article

\title{
Timoshenko Beam Theory: First-Order Analysis, Second-Order Analysis, Stability, and Vibration Analysis Using the Finite Difference Method
}

\author{
Valentin Fogang \\ Civil Engineer, C/o BUNS Sarl, P.O Box 1130, Yaounde, Cameroon; valentin.fogang@bunscameroun.com \\ ORCID iD https://orcid.org/0000-0003-1256-9862
}

\begin{abstract}
This paper presents an approach to the Timoshenko beam theory (TBT) using the finite difference method (FDM). The Timoshenko beam theory covers cases associated with small deflections based on shear deformation and rotary inertia considerations. The FDM is an approximate method for solving problems described with differential equations. It does not involve solving differential equations; equations are formulated with values at selected points of the structure. In addition, the boundary conditions and not the governing equations are applied at the beam's ends. The model developed in this paper consisted of formulating differential equations with finite differences and introducing additional points at the beam's ends and at positions of discontinuity (concentrated loads or moments, supports, hinges, springs, brutal change of stiffness, spring-mass system, etc.). The introduction of additional points allowed us to apply the governing equations at the beam's ends. Moreover, grid points with variable spacing were considered, the grid being uniform within beam segments. First-order, second-order, and vibration analyses of structures were conducted with this model. Furthermore, tapered beams were analyzed (element stiffness matrix, second-order analysis, vibration analysis). Finally, a direct time integration method (DTIM) was presented; the FDM-based DTIM enabled the analysis of forced vibration of structures, with damping taken into account. The results obtained in this paper showed good agreement with those of other studies, and the accuracy was increased through a grid refinement. Especially in the first-order analysis of uniform beams, the results were exact for uniformly distributed and concentrated loads regardless of the grid.
\end{abstract}

Keywords: Timoshenko beam; finite difference method; additional points; element stiffness matrix; tapered beam; second-order analysis; vibration analysis; direct time integration method

\section{Introduction}

This paper describes the application of Fogang's model [1] based on the finite difference method (FDM), used for the Euler-Bernoulli beam, to the Timoshenko beam. First-order analysis of the Timoshenko beam is routinely performed; the principle of virtual work yields accurate results and is easy to apply. However, second-order and vibration analyses of the Timoshenko beam cannot be modeled using the principle of virtual work. 


\section{TIMOSHENKO BEAM THEORY USING THE FINITE DIFFERENCE METHOD}

The analytical approach consists of solving the governing equations that are expressed via means of coupled partial differential equations, and satisfying the boundary and continuity conditions. Unfortunately, solving the coupled differential equations may be difficult in the presence of an axial force (or external distributed axial forces), an elastic Winkler foundation, tapered beams, or damping (by vibration analysis). Numerical methods permit therefore to overcome solving the differential equations. A considerable volume of literature has been published on numerical methods for Timoshenko beam analysis. Kindelan et al. [2] presented a method of obtaining optimal finite difference formulas that maximize their frequency range of validity. Both conventional and staggered equispaced stencils for first and second derivatives were considered. Onyia et al. [3] presented a finite element formulation to determine the critical buckling load of the unified beam element that is free from shear locking using the energy method; the technique provides a unified approach to performing stability analysis of beams with any end conditions. In stability analysis, Timoshenko and Gere [4] proposed formulas to account for shear stiffness by means of calculation of buckling loads of the associated Euler-Bernoulli beams. Hu et al. [5] used matrix structural analysis to derive a closed-form solution of the second-order element stiffness matrix; the buckling loads of single-span beams were also determined. Fogang [6] presented a relationship between curvature, bending moment, and shear force; based on this relationship closed-form expressions of efforts and deformations were derived, as well as first- and second-order element stiffness matrices. Mwabora et al. [7] considered numerical solutions for static and dynamic stability parameters of an axially loaded uniform beam resting on simply supported foundations using the FDM, where a central difference scheme was developed. Soltani et al. [8] applied the FDM to evaluate natural frequencies of non-prismatic beams with different boundary conditions and resting on variable one- or two-parameter elastic foundations. Boreyri et al. [9] analyzed the free vibration of a new type of tapered beam, with exponentially varying thickness, resting on a linear foundation; the solution was based on a semi-analytical technique, the differential transform method. Torabi et al. [10] presented an exact closed-form solution for free vibration analysis of Euler-Bernoulli conical and tapered beams carrying any desired number of attached masses; the concentrated masses were modeled by Dirac's delta functions. Fogang [11] presented a relationship between curvature, bending moment, shear force, and natural frequency; based on this relationship closedform expressions of efforts and deformations were derived, and natural frequencies were determined by solving the associated eigenvalue problem. Yesilce et al. [12] studied the free vibration of a multi-span Timoshenko beam carrying multiple spring-mass systems; natural frequencies were calculated using the secant method, and mode shapes were presented in graphs. Katsikadelis [13] presented a direct time integration method for solving the equations of motion describing the dynamic response of structural linear and nonlinear multi-degree-of-freedom systems; the method was also applied to equations with variable coefficients. Ghannadiasl [14] used Green functions to analytically solve the case of beams with various boundary conditions, resting on an elastic Winkler foundation and subjected to an axial load; the Green function method was used to evaluate the free vibration of the Timoshenko beam. Kruszewski [15] presented a theoretical analysis of the effect of transverse shear and rotary inertia on the natural frequencies of a uniform cantilevered Timoshenko beam. Soltani [16] developed a semi-analytical technique to investigate the free bending vibration behavior of an axially functionally graded non-prismatic Timoshenko beam subjected to a point force at both ends, based on the power series expansion. 


\section{TIMOSHENKO BEAM THEORY USING THE FINITE DIFFERENCE METHOD}

In the classical analysis using the FDM, points outside the beam are not considered. The boundary conditions and not the governing equations are applied at the beam's ends. Consequently, the non-application of the governing equations at the beam's ends has led to inaccurate results, making the FDM less interesting in comparison to other numerical methods such as the finite element method. This paper presented a model based on the FDM; it consisted of formulating the differential equations with finite differences and introducing additional points at the beam's ends and at positions of discontinuity (concentrated loads or moments, supports, hinges, springs, change of grid spacing, brutal change of stiffness, and spring-mass system, etc.). The introduction of additional points allowed us to apply the governing equations at the beam's ends. First-order, second-order, and vibration analyses of structures were conducted using the model. Finally, a direct time integration method (DTIM) was presented; the FDM-based DTIM enabled the analysis of forced vibration of structures, the damping being considered.

\section{Materials and methods}

\subsection{First-order analysis}

\subsubsection{Statics}

The sign convention adopted for the loads, bending moments, shear forces, and displacements is illustrated in Figure 1.
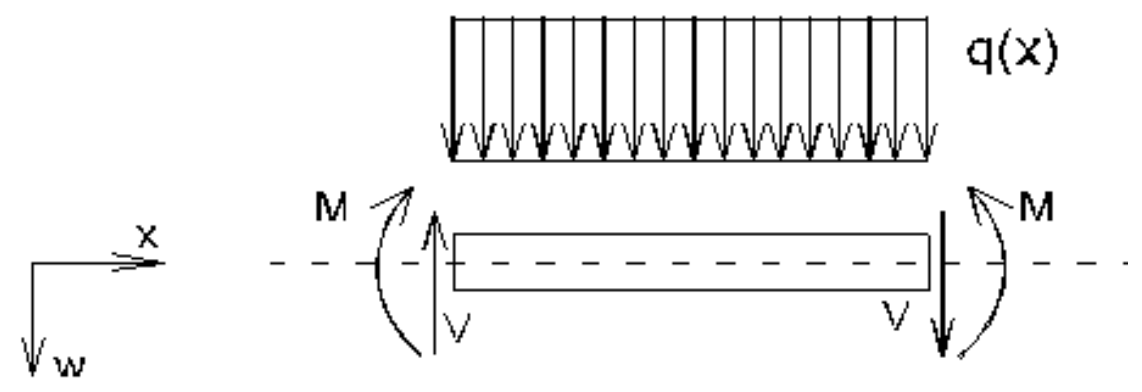

Figure 1 Sign convention for loads, bending moments, shear forces, and displacements

Specifically, $\mathrm{M}(x)$ is the bending moment in the section, $\mathrm{V}(x)$ is the shear force, $\mathrm{w}(x)$ is the deflection, and $\mathrm{q}(x)$ is the distributed load in the positive downward direction.

In first-order analysis the equations of static equilibrium on an infinitesimal element are as follows:

$$
\begin{aligned}
& \frac{d V(x)}{d x}-k(x) w(x)=-q(x) \\
& \frac{d M(x)}{d x}-V(x)=0,
\end{aligned}
$$

where $\mathrm{k}(x)$ is the stiffness of the elastic Winkler foundation. Substituting Equation (1b) into Equation (1a) yields

$$
\frac{d^{2} M(x)}{d x^{2}}-k(x) w(x)=-q(x)
$$




\section{TIMOSHENKO BEAM THEORY USING THE FINITE DIFFERENCE METHOD}

According to Timoshenko beam theory, the bending moment and shear force are related to the deflection and rotation (positive in clockwise) of the cross section $\varphi(\mathrm{x})$, as follows:

$$
M(x)=-E I \frac{d \varphi(x)}{d x} \quad \text { (3) } \quad V(x)=\kappa G A \times\left(\frac{d w(x)}{d x}-\varphi(x)\right)
$$

where $\mathrm{E}$ is the elastic modulus, $\mathrm{I}$ is the second moment of area, $\kappa$ is the shear correction factor, $\mathrm{G}$ is the shear modulus, and $\mathrm{A}$ is the cross-sectional area.

In the case of a uniform beam, substituting Equations (3) and (4) into Equations (1a) and (1b) yields

$$
\begin{aligned}
& \kappa G A \times\left(\frac{d^{2} w(x)}{d x^{2}}-\frac{d \varphi(x)}{d x}\right)-k w(x)=-q(x) \\
& E I \frac{d^{2} \varphi(x)}{d x^{2}}+\kappa G A \times\left(\frac{d w(x)}{d x}-\varphi(x)\right)=0
\end{aligned}
$$

In the case of a tapered beam, substituting Equations (3) and (4) into Equations (1a) and (1b) yields

$$
\begin{aligned}
& \frac{d \kappa G A(x)}{d x} \times\left(\frac{d w(x)}{d x}-\varphi(x)\right)+\kappa G A(x) \times\left(\frac{d^{2} w(x)}{d x^{2}}-\frac{d \varphi(x)}{d x}\right)-k w(x)=-q(x) \\
& \frac{d E I(x)}{d x} \times \frac{d \varphi(x)}{d x}+E I(x) \frac{d^{2} \varphi(x)}{d x^{2}}+\kappa G A(x) \times\left(\frac{d w(x)}{d x}-\varphi(x)\right)=0
\end{aligned}
$$

Fogang [6] presented the following relationships for a uniform beam and a tapered beam, respectively:

$$
\begin{aligned}
& \frac{d^{2} w(x)}{d x^{2}}+\frac{M(x)}{E I}-\frac{1}{\kappa G A} \frac{d^{2} M(x)}{d x^{2}}=0, \\
& \frac{d^{2} w(x)}{d x^{2}}+\frac{M(x)}{E I(x)}-\frac{1}{\kappa G A(x)} \frac{d^{2} M(x)}{d x^{2}}+\frac{1}{(\kappa G A(x))^{2}} \times \frac{d \kappa G A(x)}{d x} \times \frac{d M(x)}{d x}=0,
\end{aligned}
$$

Differentiating Equation (6c) twice with respect to $x$ and combining the result with Equation (2) yields the following widely known formula for a uniform beam without Winkler foundation:

$$
E I \frac{d^{4} w(x)}{d x^{4}}=q(x)-\frac{E I}{\kappa G A} \frac{d^{2} q(x)}{d x^{2}}
$$

In the presence of an elastic Winkler foundation, Equation (6e) becomes

$$
E I \frac{d^{4} w(x)}{d x^{4}}-\frac{E I}{\kappa G A} \frac{d^{2}(k(x) w(x))}{d x^{2}}+k(x) w(x)=q(x)-\frac{E I}{\kappa G A} \frac{d^{2} q(x)}{d x^{2}}
$$




\section{TIMOSHENKO BEAM THEORY USING THE FINITE DIFFERENCE METHOD}

For a uniform beam, the bending moment, the shear force, and the rotation of the cross section are derived using Equations (6c) and (2), Equation (1b), and Equation (4), respectively, as follows:

$$
\begin{aligned}
& M(x)=-E I \frac{d^{2} w(x)}{d x^{2}}+\frac{E I}{\kappa G A} k(x) w(x)-\frac{E I}{\kappa G A} p(x) \\
& V(x)=-E I \frac{d^{3} w(x)}{d x^{3}}+\frac{E I}{\kappa G A} \frac{d(k(x) w(x))}{d x}-\frac{E I}{\kappa G A} \frac{d p(x)}{d x} \\
& \varphi(x)=\frac{d w(x)}{d x}+\frac{E I}{\kappa G A} \frac{d^{3} w(x)}{d x^{3}}-\frac{E I}{(\kappa G A)^{2}} \frac{d(k(x) w(x))}{d x}+\frac{E I}{(\kappa G A)^{2}} \frac{d p(x)}{d x}
\end{aligned}
$$

In summary, a W- $\Phi$ finite difference approximation (FDA) using Equations (5a) to (6b), an M-W FDA using Equations (2) and (6c-d), and a W FDA using Equations (6e-i) can be considered.

\subsubsection{FDM Formulation of equations, efforts, deformations, and loadings}

\subsubsection{Fundamentals of FDM}

Figure 2 shows a segment of a beam having equidistant points with grid spacing h.

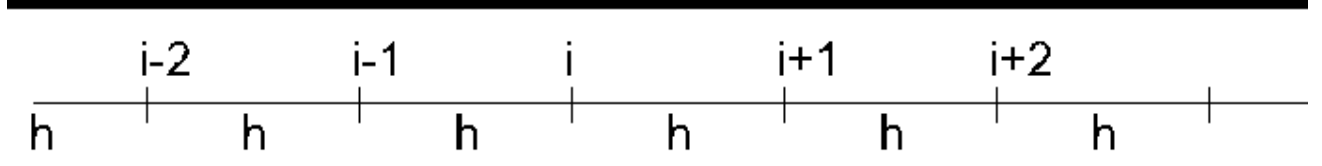

Figure 2 Beam with equidistant points

Equations (5a)-(6d) have a second-order derivative; consequently, the deflection, rotation, and moment curves $\mathrm{w}(x)$, $\varphi(x)$, and $\mathrm{M}(x)$, respectively, are approximated around the point of interest $\mathrm{i}$ as second-degree polynomials.

Thus, curves $\mathrm{w}(x)$ and $\varphi(x)$ can be described with the deflections values at equidistant grid points:

$$
w(x)=w_{i-1} \times f_{i-1}(x)+w_{i} \times f_{i}(x)+w_{i+1} \times f_{i+1}(x)
$$

The shape functions $f_{j}(x)(j=i-1, i, i+1)$ can be expressed using Lagrange polynomials:

$$
f_{j}(x)=\prod_{\substack{k=i-1 \\ k \neq j}}^{i+1} \frac{x-x_{k}}{x_{j}-x_{k}}
$$

Thus, a three-point stencil is used to write finite difference approximations to derivatives at grid points. The derivatives $(\mathrm{S}(x)$ representing $\mathrm{w}(x)$ or $\varphi(x))$ at $\mathbf{i}$ are expressed with deflection values at points $\mathrm{i}-1, \mathrm{i}$, and $\mathrm{i}+1$.

$$
\left.\frac{d^{2} S(x)}{d x^{2}}\right|_{i}=\frac{S_{i-1}-2 S_{i}+S_{i+1}}{h^{2}}
$$

$$
\left.\frac{d S(x)}{d x}\right|_{i}=\frac{-S_{i-1}+S_{i+1}}{2 h}
$$




\section{TIMOSHENKO BEAM THEORY USING THE FINITE DIFFERENCE METHOD}

Equation (6e) has a fourth-order derivative; consequently, the deflection curve is approximated around the point of interest $\mathbf{i}$ as a fourth-degree polynomial. Thus, a five-point stencil is used to write finite difference approximations to derivatives at grid points. The derivatives at $\mathbf{i}$ are expressed with deflection values at points $\mathrm{i}-2, \mathrm{i}-1, \mathrm{i}, \mathrm{i}+1$, and $\mathrm{i}+2$.

$$
\begin{aligned}
& \left.\frac{d^{4} w}{d x^{4}}\right|_{i}=\frac{w_{i-2}-4 w_{i-1}+6 w_{i}-4 w_{i+1}+w_{i+2}}{h^{4}} \\
& \left.\frac{d^{3} w}{d x^{3}}\right|_{i}=\frac{-w_{i-2}+2 w_{i-1}-2 w_{i+1}+w_{i+2}}{2 h^{3}} \\
& \left.\frac{d^{2} w}{d x^{2}}\right|_{i}=\frac{-w_{i-2}+16 w_{i-1}-30 w_{i}+16 w_{i+1}-w_{i+2}}{12 h^{2}} \\
& \left.\frac{d w}{d x}\right|_{i}=\frac{w_{i-2}-8 w_{i-1}+8 w_{i+1}-w_{i+2}}{12 h}
\end{aligned}
$$

\subsubsection{Uniform beam within segments}

\subsection{W $-\Phi$ FDA in first-order analysis of a uniform beam}

Let us consider a segment $\mathrm{k}$ of the beam (length $\mathrm{l}$ ) having equidistant grid points with spacing $\mathrm{h}_{\mathrm{k}}$. The flexural and shear stiffness values in this beam segment are $\mathrm{EI}_{\mathrm{k}}$ and $\kappa \mathrm{GA}_{\mathrm{k}} . \alpha_{\mathrm{r}}$ is the bending shear factor. A reference flexural stiffness $\mathrm{EI}_{\mathrm{r}}$ and a reference shear stiffness $\kappa \mathrm{GA}_{\mathrm{r}}$ are introduced as follows:

$$
\begin{aligned}
& E I_{k}=\beta_{M k} \times E I_{r} \\
& \kappa G A_{k}=\beta_{V k} \times \kappa G A_{r} \\
& \alpha_{r}=E I_{r} /\left(\kappa G A_{r} l^{2}\right)
\end{aligned}
$$

$$
\begin{aligned}
& h_{k}=\beta_{l k} l \\
& W(x)=E I_{r} \times w(x) \\
& \Phi(x)=E I_{r} \times \varphi(x)
\end{aligned}
$$

Substituting Equations (8a)-(9f) into Equations (5a-b) yields the following governing equations:

$$
\begin{aligned}
& \beta_{V k} W_{i-1}-\left(2 \beta_{V k}+\alpha_{r} \beta_{l k}^{2} \frac{k_{i} l^{4}}{E I_{r}}\right) W_{i}+\beta_{V k} W_{i+1}+\frac{\beta_{V k}}{2} h_{k} \Phi_{i-1}-\frac{\beta_{V k}}{2} h_{k} \Phi_{i+1}=-\frac{\alpha_{r}}{\beta_{l k}^{2}} q_{i} h_{k}^{4} \\
& -\frac{\beta_{V k} \beta_{l k}^{2}}{2 \alpha_{r}} W_{i-1}+\frac{\beta_{V k} \beta_{l k}^{2}}{2 \alpha_{r}} W_{i+1}+\beta_{M k} h_{k} \Phi_{i-1}-\left(2 \beta_{M k}+\frac{\beta_{V k} \beta_{l k}^{2}}{\alpha_{r}}\right) h_{k} \Phi_{i}+\beta_{M k} h_{k} \Phi_{i+1}=0
\end{aligned}
$$

Substituting Equations (8b) and (9a-f) into Equations (3)-(4) yields the bending moment and shear force, as follows:

$$
\begin{aligned}
& M_{i}=\beta_{M k} \frac{\Phi_{i-1}-\Phi_{i+1}}{2 h_{k}} \\
& V_{i}=\frac{\beta_{V k} \beta_{l k}^{2}}{\alpha_{r} h_{k}^{3}}\left(-\frac{W_{i-1}}{2}+\frac{W_{i+1}}{2}-h_{k} \Phi_{i}\right)
\end{aligned}
$$




\subsection{W FDA in first-order analysis of a uniform beam}

Equation (6f) is the governing equation. The stiffness $\mathrm{k}(x)$ of the Winkler foundation is assumed constant in the beam segment and is denoted by $\mathrm{k}_{\mathrm{k}}$. Substituting Equations (8c), (8e), and (9a-e) into Equation (6f) yields the following FDM formulations of the governing equation

$$
\begin{aligned}
& \beta_{M k}\left(W_{i-2}-4 W_{i-1}+6 W_{i}-4 W_{i+1}+W_{i+2}\right)+K^{*} \frac{W_{i-2}-16 W_{i-1}+30 W_{i}-16 W_{i+1}+W_{i+2}}{12} \\
& +\frac{k_{k} h_{k}^{4}}{E I_{r}} W_{i}=q_{i} h_{k}^{4}-\left.\frac{\beta_{M k} \alpha_{r}}{\beta_{V k} \beta_{l k}^{2}} h_{k}{ }^{6} \frac{d^{2} q(x)}{d x^{2}}\right|_{i} \\
& K^{*}=\frac{\beta_{M k} \alpha_{r}}{\beta_{V k} \beta_{l k}^{2}} \frac{k_{k} h_{k}^{4}}{E I_{r}} \\
& k_{k}=k_{W} \frac{E I_{r}}{l^{4}}
\end{aligned}
$$

The bending moment, the shear force, and the rotation of the cross section are calculated using Equations (6g), (8e), and (12b), Equations (6h), (8d), and (12b), and Equations (6i), (8f), and (8d) as follows:

$$
\begin{aligned}
M_{i}=\beta_{M K} \frac{W_{i-2}-16 W_{i-1}+30 W_{i}-16 W_{i+1}+W_{i+2}}{12 h_{k}^{2}}+K^{*} \frac{W_{i}}{h_{k}^{2}}-\frac{\beta_{M k} \alpha_{r}}{\beta_{V k}} l^{2} p_{i} \\
V_{i}=\beta_{M K} \frac{W_{i-2}-2 W_{i-1}+2 W_{i+1}-W_{i+2}}{2 h_{k}^{3}}+K^{*} \frac{W_{i-2}-8 W_{i-1}+8 W_{i+1}-W_{i+2}}{12 h_{k}^{3}}-\left.\frac{\beta_{M k} \alpha_{r}}{\beta_{V k}} l^{2} \frac{d p(x)}{d x}\right|_{i} \\
E I_{r} \varphi(x)= \\
\quad\left(1-\frac{\beta_{M k} k_{W} \alpha_{r}^{2}}{\beta_{V k}^{2}}\right) \frac{W_{i-2}-8 W_{i-1}+8 W_{i+1}-W_{i+2}}{12 h_{k}} \\
+\frac{\beta_{M k} \alpha_{r}}{\beta_{V k} \beta_{l k}^{2}} \frac{-W_{i-2}+2 W_{i-1}-2 W_{i+1}+W_{i+2}}{2 h_{k}}+\left.\frac{\beta_{M k} \alpha_{r}^{2}}{\beta_{V k}^{2}} l^{4} \frac{d p(x)}{d x}\right|_{i}
\end{aligned}
$$

\subsection{M-W FDA in first-order analysis of a uniform beam}

Equations (2) and (6c) are the governing equations. Applying Equations (8a-b) and (9a-e) in Equations (2) and (6c) yields the corresponding FDM expressions, as follows:

$$
\begin{aligned}
& h_{k}^{2} M_{i-1}-2 h_{k}^{2} M_{i}+h_{k}^{2} M_{i+1}-\beta_{l k}^{4} \frac{k_{i} l^{4}}{E I_{r}} W_{i}=-q_{i} h_{k}^{4} \\
& \beta_{M k}\left(W_{i-1}-2 W_{i}+W_{i+1}\right)-\frac{\beta_{M k} \alpha_{r}}{\beta_{V k} \beta_{l k}^{2}}\left(h_{k}^{2} M_{i-1}-2 h_{k}^{2} M_{i}+h_{k}^{2} M_{i+1}\right)+h_{k}^{2} M_{i}=0
\end{aligned}
$$


The shear force (Equations (1b) and (8b)) and the rotation of the cross section (Equations (1b), (4), (8b), and (9b-e)) are calculated as follows:

$V_{i}=\frac{M_{i+1}-M_{i-1}}{2 h_{k}}$ (13f) $\quad E I_{r} \times \varphi_{i}=\frac{W_{i+1}-W_{i-1}}{2 h_{k}}-\frac{\alpha_{r}}{2 h_{k} \beta_{V k} \beta_{l k}^{2}}\left(h_{k}^{2} M_{i+1}-h_{k}^{2} M_{i-1}\right)$

\subsubsection{Tapered beam within segments}

\subsection{W $-\Phi$ FDA in first-order analysis of a tapered beam}

The following parameters describing the stiffnesses $\operatorname{EI}(x)$ and $\kappa \mathrm{GA}(x)$ and their rate of change are defined.

$$
\begin{array}{lll}
E I(x)=\beta_{M}(x) \times E I_{r} & (14 \mathrm{a}) & \beta_{M}^{\prime}(x)=h_{k} \frac{d \beta_{M}(x)}{d x} \\
\kappa G A(x)=\beta_{V}(x) \times \kappa G A_{r} & (14 \mathrm{~b}) & \beta_{V}^{\prime}(x)=h_{k} \frac{d \beta_{V}(x)}{d x}
\end{array}
$$

Substituting Equations (9d) and (14a-d) into Equations (6a-b) yields the following governing equations for the tapered beam at position $\mathbf{i}$ :

$$
\begin{aligned}
& \left(-\frac{\beta_{V i}^{\prime}}{2}+\beta_{V i}\right) W_{i-1}-\left(2 \beta_{V i}+\alpha_{r} \beta_{l k}^{2} \frac{k_{i} l^{4}}{E I_{r}}\right) W_{i}+\left(\frac{\beta_{V i}^{\prime}}{2}+\beta_{V i}\right) W_{i+1} \\
& +\frac{\beta_{V i}}{2} h_{k} \Phi_{i-1}-\beta_{V i}^{\prime} h_{k} \Phi_{i}-\frac{\beta_{V i}}{2} h_{k} \Phi_{i+1}=-\frac{\alpha_{r}}{\beta_{l k}^{2}} q_{i} h_{k}^{4} \\
& -\frac{\beta_{V i} \beta_{l k}^{2}}{2 \alpha_{r}} W_{i-1}+\frac{\beta_{V i} \beta_{l k}^{2}}{2 \alpha_{r}} W_{i+1}+\left(\beta_{M i}-\frac{\beta_{M i}^{\prime}}{2}\right) h_{k} \Phi_{i-1}-\left(2 \beta_{M i}+\frac{\beta_{V i} \beta_{l k}^{2}}{\alpha_{r}}\right) h_{k} \Phi_{i} \\
& +\left(\frac{\beta_{M i}^{\prime}}{2}+\beta_{M i}\right) h_{k} \Phi_{i+1}=0
\end{aligned}
$$

The bending moment and shear force are calculated using Equations (11a-b), $\beta_{\mathrm{Mk}}$ and $\beta_{\mathrm{Vk}}$ being replaced by $\beta_{\mathrm{Mi}}$ and $\beta_{\mathrm{Vi}}$

\subsection{M-W FDA in first-order analysis of a tapered beam}

Equations (2) and (6d) are the governing equations. Substituting Equations (8a-b), (9d), and (14a-d) into Equations (2) and (6d) yields Equation (13d) and the following equation:

$$
\begin{aligned}
& \beta_{M i} W_{i-1}-2 \beta_{M i} W_{i}+\beta_{M i} W_{i+1}-\frac{\beta_{M i} \alpha_{r}}{\beta_{V i} \beta_{l k}^{2}}\left(1+\frac{\beta_{V i}^{\prime}}{2 \beta_{V i}}\right) h_{k}^{2} M_{i-1}+\left(1+\frac{2 \beta_{M i} \alpha_{r}}{\beta_{V i} \beta_{l k}^{2}}\right) h_{k}^{2} M_{i} \\
& +\frac{\beta_{M i} \alpha_{r}}{\beta_{V i} \beta_{l k}^{2}}\left(\frac{\beta_{V i}^{\prime}}{2 \beta_{V i}}-1\right) h_{k}^{2} M_{i+1}=0
\end{aligned}
$$




\section{TIMOSHENKO BEAM THEORY USING THE FINITE DIFFERENCE METHOD}

Thus, Equations (13d) and (16) are the governing equations. The shear force and the rotation of the cross section are calculated using Equations (13f) and (13g), respectively. However, $\beta_{\mathrm{Vk}}$ is replaced by $\beta_{\mathrm{Vi}}$ in Equation (13g).

\subsubsection{FDA of $\mathrm{q}(x)$ and $\mathrm{k}(x)$}

Fogang [1] presented formulas to determine FDA of distributed loads and stiffness of an elastic Winkler foundation. The FDA $q_{i}$ for position i being the left beam's end, an interior point on the beam, or the right beam's end was as follows:

$$
\begin{aligned}
& q_{i}=\frac{1}{2 h}\left[3 \int_{i}^{i+1} q(x) d x-\int_{i+1}^{i+2} q(x) d x\right] \\
& q_{i}=\frac{1}{2 h} \int_{i-1}^{i+1} q(x) d x \\
& q_{i}=\frac{1}{2 h}\left[-\int_{i-2}^{i-1} q(x) d x+3 \int_{i-1}^{i} q(x) d x\right]
\end{aligned}
$$

The application of Equations (17a) to (18) shows that for a linearly distributed load, $\mathrm{q}_{\mathrm{i}}=\mathrm{q}\left(\mathrm{x}_{\mathrm{i}}\right)$.

At any point i, the stiffness of the elastic Winkler foundation, $\mathrm{ki}$, is calculated similarly to Equations (17a) to (18).

\subsubsection{Analysis at positions of discontinuity}

Positions of discontinuity are positions of application of concentrated external loads (force or moment), supports, hinges, springs, abrupt change in cross section, positions where $\mathrm{EI}(x)$ and $\kappa \mathrm{GA}(x)$ are not differentiable, and change in grid spacing.

\subsubsection{Uniform beam within segments}

Concentrated loads (force $\mathrm{P}$ and moment $\mathrm{M}^{*}$ ) are applied at point i, as shown in Figure 3. The beam has a uniform cross section within segments; at point $\mathrm{i}$, an abrupt change in cross section and a change in grid spacing are assumed.
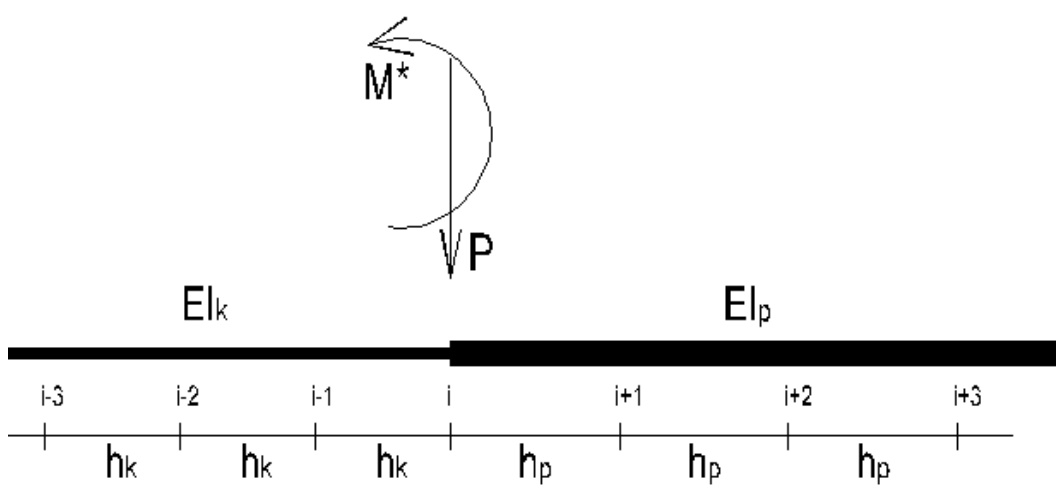

Figure 3 Beam at a position of discontinuity

Fogang's [1] model consists of realizing an opening of the beam at point $i$ and introducing additional points in the opening, as represented in Figure 4a,b and Figure 5a,b. 


\subsection{W $-\Phi$ FDA of a uniform beam}

Figure 4a,b below shows the additional points (points ia, id) introduced in the opening. The unknowns at any point are the deflection and the rotation of the cross section.
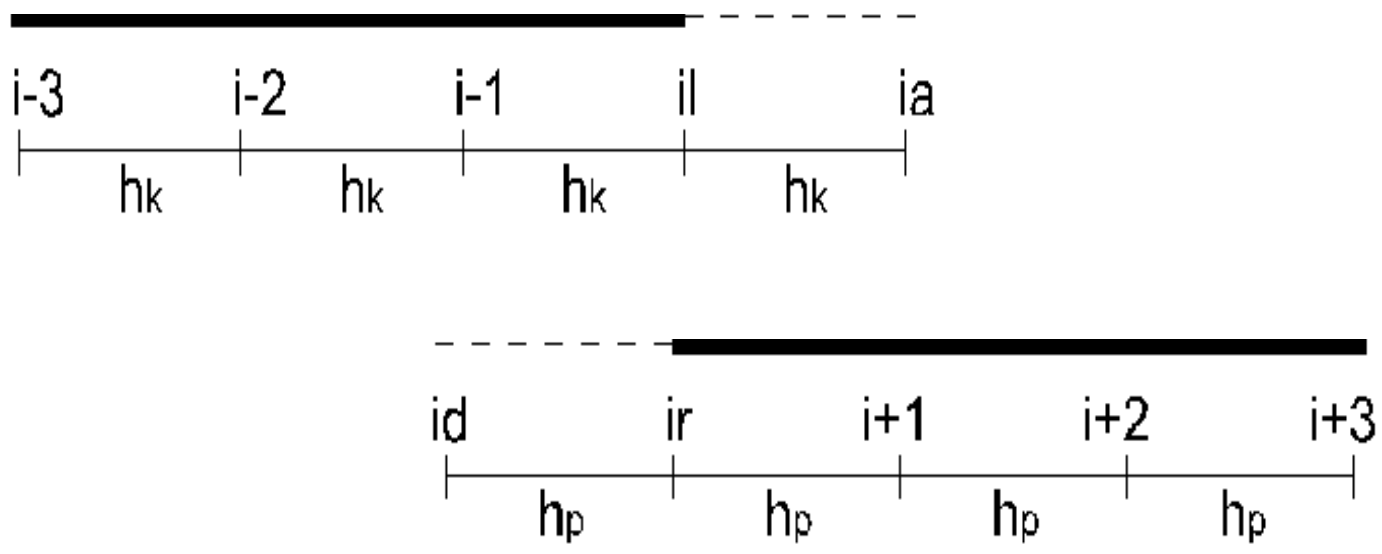

Figure 4 Opening of the beam and introduction of additional points on the left side (a) and the right side (b)

The governing equations (Equations (10a-b)) are applied at any point of the beam: ...i-1, il, ir, i+1 ...

Thus, the governing equations at position il are as follows:

$\beta_{V k} W_{i-1}-\left(2 \beta_{V k}+\alpha_{r} \beta_{l k}^{2} \frac{k_{i} l^{4}}{E I_{r}}\right) W_{i l}+\beta_{V k} W_{i a}+\frac{\beta_{V k}}{2} h_{k} \Phi_{i-1}-\frac{\beta_{V k}}{2} h_{k} \Phi_{i a}=-\frac{\alpha_{r}}{\beta_{l k}^{2}} q_{i l} h_{k}^{4}$

$-\frac{\beta_{V k} \beta_{l k}^{2}}{2 \alpha_{r}} W_{i-1}+\frac{\beta_{V k} \beta_{l k}^{2}}{2 \alpha_{r}} W_{i a}+\beta_{M k} h_{k} \Phi_{i-1}-\left(2 \beta_{M k}+\frac{\beta_{V k} \beta_{l k}^{2}}{\alpha_{r}}\right) h_{k} \Phi_{i l}+\beta_{M k} h_{k} \Phi_{i a}=0$

Similarly, the governing equations at position ir are formulated. The continuity equations express the continuity of the deflection and rotation of the cross section, and the equilibrium of bending moment and shear force (Equations (11a-b)):

$$
\begin{aligned}
& w_{i l}=w_{i r} \rightarrow W_{i l}=W_{i r} \\
& \varphi_{i l}=\varphi_{i r} \rightarrow \Phi_{i l}=\Phi_{i r} \\
& M_{i l}-M_{i r}=M^{*} \rightarrow \beta_{M k} \frac{\Phi_{i-1}-\Phi_{i a}}{2 h_{k}}-\beta_{M p} \frac{\Phi_{i d}-\Phi_{i+1}}{2 h_{p}}=M^{*} \\
& V_{i l}-V_{i r}=P \\
& \rightarrow \frac{\beta_{V k} \beta_{l k}^{2}}{\alpha_{r} h_{k}^{3}}\left(-\frac{W_{i-1}}{2}+\frac{W_{i a}}{2}-h_{k} \Phi_{i l}\right)-\frac{\beta_{V p} \beta_{l p}^{2}}{\alpha_{r} h_{p}^{3}}\left(-\frac{W_{i d}}{2}+\frac{W_{i+1}}{2}-h_{p} \Phi_{i r}\right)=P
\end{aligned}
$$

An adjustment of the continuity equations is made in the case of a hinge (no continuity of the rotation of the cross section, and $\mathrm{M}_{\mathrm{il}}=\mathrm{M}_{\mathrm{ir}}=0$ ), a support $\left(\mathrm{W}_{\mathrm{il}}=\mathrm{W}_{\mathrm{ir}}=0\right.$, no Equation (20d)), or a spring.

At the beam's ends, additional points are introduced (Figure 4a,b), so governing equations are applied at the beam's ends, as well as boundary conditions. 


\subsection{W FDA of a uniform beam}

Figure 5a,b below shows the additional points (fictive points ia, ib, ic, id) introduced in the opening. The unknown at any point is the deflection.
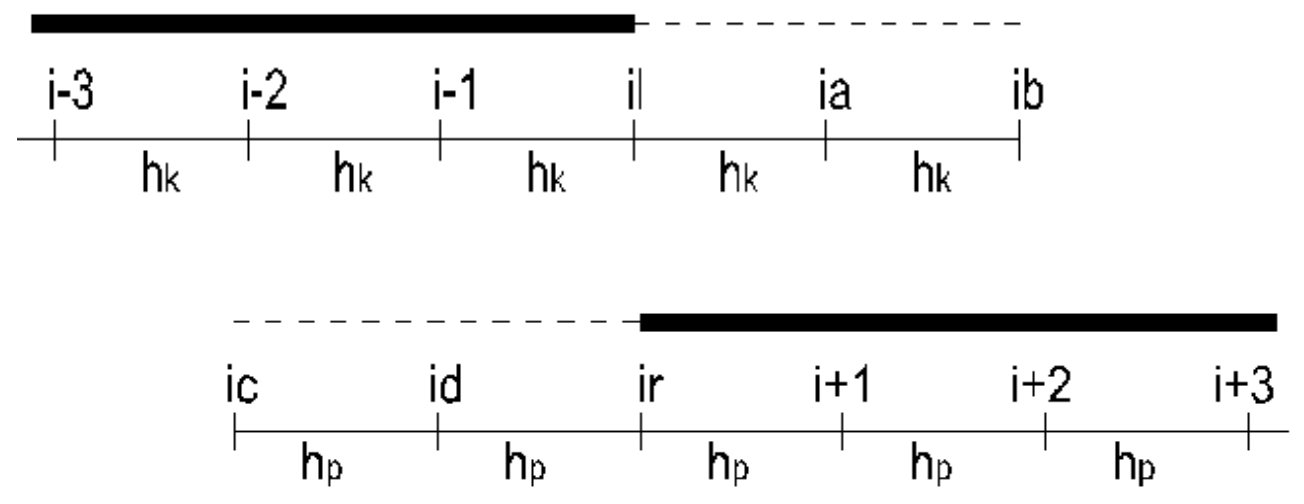

Figure 5 Opening of the beam and introduction of additional points on the left side (a) and the right side (b)

The governing equation (Equation (12a)) is applied at any point of the beam: ... i-2, i-1, il, ir, i+1, i+2... Thus, the governing equation (Equation (12a)) at position il is formulated by adopting for i, i+1, and i+2 the values of il, ia, and $\mathrm{ib}$, respectively. Similarly, the governing equation at position ir is formulated by adopting for i, i-1, and i-2 the values of ir, id, and ic, respectively. The continuity equations can be expressed using Equations (13a-c), as follows:

$$
\begin{aligned}
& w_{i l}=w_{i r} \rightarrow W_{i l}=W_{i r} \\
& \varphi_{i l}=\varphi_{i r} \rightarrow E I_{r} \times \varphi_{i l}=E I_{r} \times \varphi_{i r} \\
& M_{i l}-M_{i r}=M^{*} \\
& V_{i l}-V_{i r}=P
\end{aligned}
$$

In the equations above, $\varphi_{\mathrm{il}}, \mathrm{M}_{\mathrm{il}}$, and $\mathrm{V}_{\mathrm{il}}$ are formulated by adopting for $\mathrm{i}, \mathrm{i}+1$, and $\mathrm{i}+2$ the values of $\mathrm{il}$, ia, and $\mathrm{ib}$, respectively. Similarly, $\varphi_{\mathrm{ir}}, \mathrm{M}_{\mathrm{ir}}$, and $\mathrm{V}_{\mathrm{ir}}$ are formulated by adopting for i, i-1, and i-2 the values of ir, id, and ic, respectively.

\subsection{M-W FDA of a uniform beam}

The additional points of Figure $4 \mathrm{a}, \mathrm{b}$ are introduced. The unknowns at any point are the deflection and the bending moment. The governing equations (Equations (13d-e)) are applied at any point of the beam: ... i-1, il, ir, i+1 ... The continuity equations can be expressed using Equations (21a-d); the shear force and the rotation of the cross section are calculated using Equations (13f) and (13g), respectively.

\subsection{Mixed FDA of a uniform beam}

Different approximations ( $\mathrm{W}-\Phi, \mathrm{W}$, and $\mathrm{M}-\mathrm{W}$ ) can be considered on either side of the point of discontinuity. The continuity equations are then formulated with the corresponding formulas. 


\subsubsection{Tapered beam within segments}

As described in Section 2.1.3.1, an opening of the beam is realized at point $\mathrm{i}$ and additional points (fictive points ia, id) are introduced in the opening (Figure 4a,b).

\subsection{W $\quad$ W FDA of a tapered beam}

The governing equations (Equations (15a-b)) are applied at any point of the beam: ... i-1, il, ir, i+1 ... The continuity equations can be expressed through an adjustment of Equations (20a-d), as follows:

$$
\beta_{M k}=\beta_{M i l} \quad \beta_{M p}=\beta_{M i r} \quad \beta_{V k}=\beta_{V i l} \quad \beta_{V p}=\beta_{V i r}
$$

\subsection{M-W FDA of a tapered beam}

The governing equations (Equations (13d) and (16)) are applied at any point of the beam: ... i-1, il, ir, i+1 ... The continuity equations can be expressed using Equations (21a-d), whereby the shear force and the rotation of the cross section are calculated using Equations (13f) and (13g), respectively. However, $\beta_{\mathrm{Vk}}$ is replaced by $\beta_{\mathrm{Vi}}$ in Equation (13g).

\subsection{Mixed FDA of a tapered beam}

Similar to the uniform beam, different approximations $(\mathrm{W}-\Phi, \mathrm{M}-\mathrm{W})$ can be considered on either side of the point of discontinuity. The continuity equations are then formulated with the corresponding formulas.

\subsubsection{Non-uniform grid}

The grid may be such that every node has a non-constant distance from another (Figure 6).

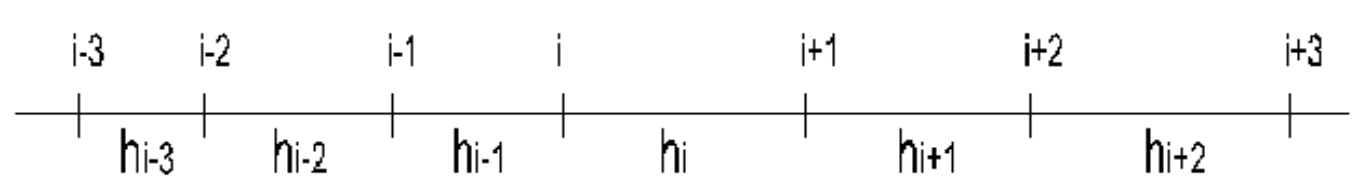

Figure 6 Beam with a non-uniform grid

In this case, the Lagrange interpolation polynomial (Equation (7b)) was used for FDM formulations. The resulting equations were complicated, so, the non-uniform grid was not further analyzed. In fact, it should not be analyzed as a discontinuity position.

\subsubsection{First-order element stiffness matrix of a tapered beam}

\subsubsection{1 $4 \times 4$ element stiffness matrix}

The sign convention for bending moments, shear forces, displacements, and rotations of the cross section adopted to determine the element stiffness matrix in local coordinates is illustrated in Figure 7. 


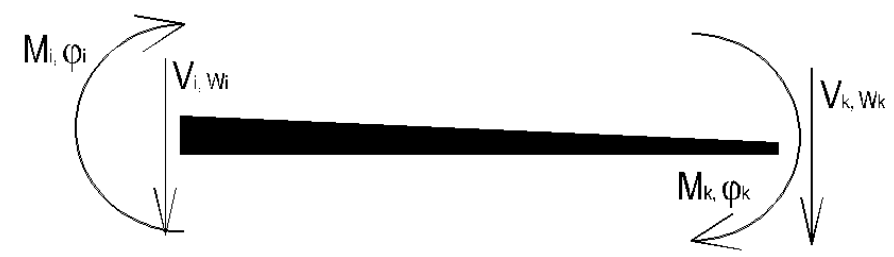

Figure 7 Sign convention for moments, shear forces, displacements, and rotations for the stiffness matrix

Let us define the following vectors:

$$
\begin{aligned}
& \overrightarrow{S_{\text {red }}}=\left[V_{i} ; M_{i} ; V_{k} ; M_{k}\right]^{T} \\
& \overrightarrow{V_{\text {red }}}=\left[w_{i} ; \varphi_{i} ; w_{k} ; \varphi_{k}\right]^{T}
\end{aligned}
$$

The $4 \times 4$ element stiffness matrix in local coordinates of the tapered beam is denoted by $\mathrm{K}_{44}$.

The vectors defined are related together with the element stiffness matrix $\mathrm{K}_{44}$, as follows:

$$
\overrightarrow{S_{\text {red }}}=K_{44} \times \overrightarrow{V_{\text {red }}}
$$

Let us divide the beam in $\mathrm{n}$ parts of equal length $\mathrm{h}\left(\mathrm{l}=\mathrm{nh}_{\mathrm{k}}\right)$, as shown in Figure 8.
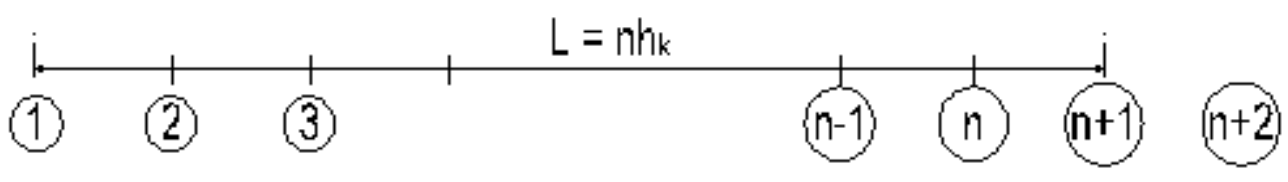

Figure 8 Finite difference method (FDM) discretization for $4 \times 4$ element stiffness matrix

\subsection{W $\quad \Phi$ FDA for element stiffness matrix of a tapered beam}

Equations (15a-b) with $\mathrm{qi}=0$ and $\mathrm{ki}=0$ are applied at any point on the grid (nodes $1,2, \ldots \mathrm{n}+1$ of Figure 8 ). Considering the sign convention adopted for bending moments and shear forces in general (Figure 1) and in the element stiffness matrix (Figure 7), the following static compatibility boundary conditions can be set in combination with Equations (11a-b):

$$
\begin{aligned}
& V_{i}=-V_{1}=-\frac{\beta_{V 1} \beta_{l k}^{2}}{\alpha_{r} h_{k}^{3}}\left(-\frac{W_{0}}{2}+\frac{W_{2}}{2}-h_{k} \Phi_{1}\right) \rightarrow \frac{\alpha_{r} h_{k}^{3}}{\beta_{V 1} \beta_{l k}^{2}} V_{i}-\frac{W_{0}}{2}+\frac{W_{2}}{2}-h_{k} \Phi_{1}=0 \\
& M_{i}=M_{1}=\beta_{M 1} \frac{\Phi_{0}-\Phi_{2}}{2 h_{k}} \rightarrow \frac{2 h_{k}^{2}}{\beta_{M 1}} M_{i}-h_{k} \Phi_{0}+h_{k} \Phi_{2}=0 \\
& V_{k}=V_{n+1}=\frac{\beta_{V n+1} \beta_{l k}^{2}}{\alpha_{r} h_{k}^{3}}\left(-\frac{W_{n}}{2}+\frac{W_{n+2}}{2}-h_{k} \Phi_{n+1}\right) \rightarrow \frac{\alpha_{r} h_{k}^{3}}{\beta_{V n+1} \beta_{l k}^{2}} V_{k}+\frac{W_{n}}{2}-\frac{W_{n+2}}{2}+h_{k} \Phi_{n+1 n}=0 \\
& M_{k}=-M_{n+1}=-\beta_{M n+1} \frac{\Phi_{n}-\Phi_{n+2}}{2 h_{k}} \rightarrow \frac{2 h_{k}^{2}}{\beta_{M n+1}} M_{k}+h_{k} \Phi_{n}-h_{k} \Phi_{n+2}=0
\end{aligned}
$$




\section{TIMOSHENKO BEAM THEORY USING THE FINITE DIFFERENCE METHOD}

Considering the sign convention adopted for displacements and rotations of cross sections in general (Figure 1) and in the element stiffness matrix (Figure 7), the following geometric compatibility boundary conditions can be set:

$$
\begin{array}{lll}
w_{1}=w_{i} \rightarrow W_{1}=E I_{r} \times w_{i} & \text { (26a) } & w_{n+1}=w_{k} \rightarrow W_{n+1}=E I_{r} \times w_{k} \\
\varphi_{1}=\varphi_{i} \rightarrow \Phi_{1}=E I_{r} \times \varphi_{i} & \text { (26b) } & \varphi_{n+1}=\varphi_{k} \rightarrow \Phi_{n+1}=E I_{r} \times \varphi_{k}
\end{array}
$$

The number of equations is $2(n+1)+4+4=\mathbf{2 n}+\mathbf{1 0}$. The number of unknowns is $2(n+3)+4=\mathbf{2 n}+\mathbf{1 0}$, especially $2(\mathrm{n}+3)$ unknowns $(\mathrm{W} ; \Phi)$ at points on the beam and additional points at the beam's ends, and four efforts at the beam's ends $\left(\mathrm{V}_{\mathrm{i}} ; \mathrm{M}_{\mathrm{i}} ; \mathrm{V}_{\mathrm{k}} ; \mathrm{M}_{\mathrm{k}}\right.$ ). Let us define the following vector

$$
\vec{S}_{1}=\left[W_{0} ; \Phi_{0} ; W_{1} ; \Phi_{1} \ldots \ldots W_{n+2} ; \Phi_{n+2}\right]^{T}
$$

The combination of Equations (15a-b) applied at any point on the grid, Equations (25a-d), and Equations (26a-d) can be expressed with matrix notation as follows, the geometric compatibility boundary conditions (Equations (26a-d)) being at the bottom:

$$
T \times\left[\begin{array}{c}
\vec{S}_{1} \\
\vec{S}_{\text {red }}
\end{array}\right]=\left[\begin{array}{c}
\overrightarrow{0} \\
E I_{r} \times \vec{V}_{\text {red }}
\end{array}\right] \rightarrow\left[\begin{array}{c}
\vec{S}_{1} \\
\vec{S}_{\text {red }}
\end{array}\right]=T^{-1} \times\left[\begin{array}{c}
\overrightarrow{0} \\
E I_{r} \times \vec{V}_{\text {red }}
\end{array}\right]
$$

The matrix $\mathbf{T}$ has $2 \mathrm{n}+10$ rows and $2 \mathrm{n}+10$ columns. The zero vector above has $2 \mathrm{n}+6$ rows.

$$
T^{-1}=\left[\begin{array}{ll}
T_{a a} & T_{a b} \\
T_{b a} & T_{b b}
\end{array}\right]
$$

The matrix $T$ aa has $2 n+6$ rows and $2 n+6$ columns, the matrix $T$ ab has $2 n+6$ rows and 4 columns, the matrix Tba has 4 rows and $2 n+6$ columns, and the matrix Tbb has 4 rows and 4 columns.

The combination of Equations (24), (28), and (29) yields the element stiffness matrix of the beam.

$$
K_{44}=E I_{r} \times T_{b b}
$$

A general matrix formulation of $\mathrm{K}_{44}$ is as follows:

$$
K_{44}=E I_{r} \times\left[\begin{array}{ll}
0 & I
\end{array}\right] \times T^{-1} \times\left[\begin{array}{c}
0^{T} \\
I
\end{array}\right]
$$

In Equation (30b), 0 is a zero matrix with 4 rows and $2 n+6$ columns, and $I$ is the $4 \times 4$ identity matrix. 


\subsection{M-W FDA for element stiffness matrix of a tapered beam}

Equations (13d) and (16) with $\mathrm{qi}=0$ and $\mathrm{ki}=0$ are applied at any point on the grid (nodes $1,2, \ldots \mathrm{n}+1$ of Figure 8). The static compatibility boundary conditions in combination with Equations (1b) and (8b) can be expressed as follows:

$$
\begin{aligned}
& V_{i}=-V_{1}=-\left.\frac{d M(x)}{d x}\right|_{1}=-\frac{M_{2}-M_{0}}{2 h_{k}} \rightarrow 2 h_{k} V_{i}+M_{2}-M_{0}=0 \\
& M_{i}=M_{1} \rightarrow M_{i}-M_{1}=0 \\
& V_{k}=V_{n+1}=\left.\frac{d M(x)}{d x}\right|_{n+1}=\frac{M_{n+2}-M_{n}}{2 h_{k}} \rightarrow 2 h_{k} V_{k}-M_{n+2}+M_{n}=0 \\
& M_{k}=-M_{n+1} \rightarrow M_{k}+M_{n+1}=0
\end{aligned}
$$

The geometric compatibility boundary conditions in combination with Equations (16d) are

$$
\begin{aligned}
& w_{1}=w_{i} \rightarrow W_{1}=E I_{r} \times w_{i} \\
& \varphi_{1}=\varphi_{i} \rightarrow E I_{r} \times \varphi_{1}=\frac{W_{2}-W_{0}}{2 h_{k}}-\frac{\alpha_{r}}{2 h_{k} \beta_{V 1} \beta_{l k}^{2}}\left(h_{k}^{2} M_{2}-h_{k}^{2} M_{0}\right)=E I_{r} \times \varphi_{i} \\
& w_{n+1}=w_{k} \rightarrow W_{n+1}=E I_{r} \times w_{k} \\
& \varphi_{n+1}=\varphi_{k} \rightarrow E I_{r} \times \varphi_{n+1}=\frac{W_{n+2}-W_{n}}{2 h_{k}}-\frac{\alpha_{r}}{2 h_{k} \beta_{V n+1} \beta_{l k}^{2}}\left(h_{k}^{2} M_{n+2}-h_{k}^{2} M_{n}\right)=E I_{r} \times \varphi_{k}
\end{aligned}
$$

The analysis continues similarly to Section 2.1.4.1.1 (Equations (27)-(30b)).

\subsubsection{2 $3 \times 3$ element stiffness matrix}

Assuming the presence of a hinge at the right end, the sign convention for bending moments, shear forces, displacements, and rotations of the cross section is illustrated in Figure 9.

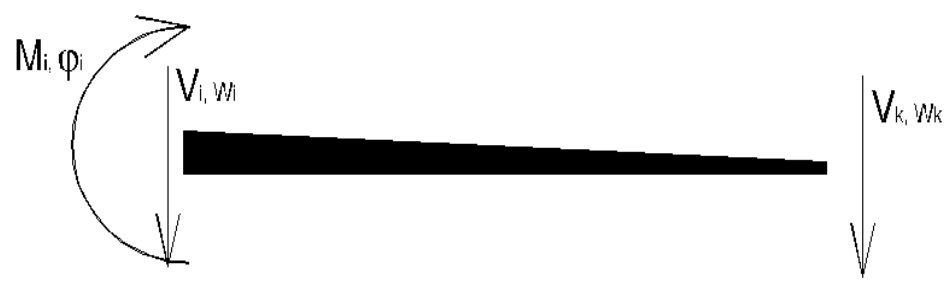

Figure 9 Sign convention for moments, shear forces, displacements, and rotations for the stiffness matrix

The $3 \times 3$ element stiffness matrix in local coordinates of the tapered beam is denoted by $\mathrm{K}_{33}$. 


\section{TIMOSHENKO BEAM THEORY USING THE FINITE DIFFERENCE METHOD}

The vectors of Equations (23a-b) and (24) become

$$
\begin{aligned}
& \overrightarrow{S_{\text {red }}}=\left[V_{i} ; M_{i} ; V_{k}\right]^{T} \\
& \overrightarrow{V_{\text {red }}}=\left[w_{i} ; \varphi_{i} ; w_{k}\right]^{T} \\
& \overrightarrow{S_{\text {red }}}=K_{33} \times \overrightarrow{V_{\text {red }}}
\end{aligned}
$$

The matrix $\mathrm{K}_{33}$ can be formulated with the values of the matrix $\mathrm{K}_{44}$ (see Equations (30a-b)).

$$
K_{44}=\left[\begin{array}{ll}
K_{a a} & K_{a b} \\
K_{b a} & K_{b b}
\end{array}\right]
$$

The matrix $\mathrm{K}_{44}$ has 4 rows and 4 columns, the matrix $\mathrm{K}_{\text {aa }}$ has 3 rows and 3 columns, the matrix $\mathrm{K}_{\mathrm{ab}}$ has 3 rows and 1 column, the matrix Kba has 1 row and 3 columns, and the matrix Kbb has 1 row and 1 column (a single value). The combination of Equation (24) with the presence of a hinge at position $\mathrm{k}\left(\mathrm{M}_{\mathrm{k}}=0\right)$ and Equation (32c) yields the matrix $\mathrm{K}_{33}$, as follows:

$$
K_{33}=K_{a a}-K_{a b} \times \frac{1}{K_{b b}} \times K_{b a}
$$

\subsection{Second-order analysis}

The equation of static equilibrium can be expressed as follows:

$$
\begin{aligned}
& \frac{d T(x)}{d x}-k(x) w(x)=-q(x) \\
& \frac{d M(x)}{d x}-T(x)+N(x) \frac{d w(x)}{d x}=0
\end{aligned}
$$

The axial force (positive in tension) is denoted by $\mathrm{N}(x)$ and the transverse force by $\mathrm{T}(x)$. Let us consider an external distributed axial load $\mathrm{n}(x)$ positive along the $+\mathrm{x}$ axis

$$
n(x)=-\frac{d N(x)}{d x}
$$

The transverse force $\mathrm{T}(x)$ is related to the shear force $\mathrm{V}(x)$, as follows:

$$
T(x)=V(x)+N(x) \frac{d w(x)}{d x}
$$




\subsubsection{Second-order analysis of a uniform beam within segments}

The grid spacing $h_{k}$, the reference flexural rigidity $E I_{r}$, the reference shear stiffness $\kappa G A_{r}$, and the parameters $\beta_{\mathrm{lk}}, \beta_{M k}$, $\beta_{\mathrm{Vk}}$, and $\alpha_{\mathrm{r}}$ are defined similarly to previous sections.

\subsubsection{W $-\Phi$ FDA in second-order analysis of a uniform beam}

Substituting Equations (4), (36), and (37) into Equation (35a) yields

$$
\kappa G A \times\left(\frac{d^{2} w(x)}{d x^{2}}-\frac{d \varphi(x)}{d x}\right)+N(x) \frac{d^{2} w(x)}{d x^{2}}-n(x) \frac{d w(x)}{d x}-k w(x)=-q(x)
$$

Substituting Equation (37) into Equation (35b) yields Equation (1b). Substituting Equations (3) and (4) into Equation (1b) yields Equation (5b). Substituting Equations (8a-b) and (9a-f) into Equations (5b) and (38) yields Equation (10b) and the following equation:

$$
\begin{aligned}
& \left(\frac{\beta_{V k} \beta_{l k}^{2}}{\alpha_{r}}+\frac{N_{i} h_{k}^{2}}{E I_{r}}+\frac{n_{i} h_{k}^{3}}{2 E I_{r}}\right) W_{i-1}-\left(2 \frac{\beta_{V k} \beta_{l k}^{2}}{\alpha_{r}}+\frac{2 N_{i} h_{k}^{2}}{E I_{r}}+\beta_{l k}^{4} \frac{k_{i} l^{4}}{E I_{r}}\right) W_{i}+\left(\frac{\beta_{V k} \beta_{l k}^{2}}{\alpha_{r}}+\frac{N_{i} h_{k}^{2}}{E I_{r}}-\frac{n_{i} h_{k}^{3}}{2 E I_{r}}\right) W_{i+1} \\
& +\frac{\beta_{V k} \beta_{l k}^{2}}{2 \alpha_{r}} h_{k} \Phi_{i-1}-\frac{\beta_{V k} \beta_{l k}^{2}}{2 \alpha_{r}} h_{k} \Phi_{i+1}=-q_{i} h_{k}^{4}
\end{aligned}
$$

Equations (10b) and (39) are applied at any point on the grid. At point $\mathrm{i}$, the external distributed axial load $\mathrm{n}_{\mathrm{i}}$ is calculated similarly to Equations (17a-c). Applying Equations (8b) and (11b) into Equation (37) yields the FDM formulation of the transverse force:

$$
T_{i}=-\frac{1}{h_{k}^{3}}\left(\frac{\beta_{V k} \beta_{l k}^{2}}{2 \alpha_{r}}+\frac{N_{i} h_{k}^{2}}{2 E I_{r}}\right) W_{i-1}+\frac{1}{h_{k}^{3}}\left(\frac{\beta_{V k} \beta_{l k}^{2}}{2 \alpha_{r}}+\frac{N_{i} h_{k}^{2}}{2 E I_{r}}\right) W_{i+1}-\frac{1}{h_{k}^{3}} \frac{\beta_{V k} \beta_{l k}^{2}}{\alpha_{r}} h_{k} \Phi_{i}
$$

The bending moment is calculated using Equation (11a). The analysis at positions of discontinuity is conducted similarly to the first-order analysis; however, the shear force is replaced by the transverse force.

\subsubsection{W FDA in second-order analysis of a uniform beam}

It is assumed here that the axial force and stiffness of the Winkler foundation are constant along the beam. Substituting Equations (2), (36), and (37) into Equation (35a) yields

$$
\frac{d^{2} M(x)}{d x^{2}}+N \frac{d^{2} w(x)}{d x^{2}}-k w(x)=-q(x)
$$

Equation (6c) also holds in second-order analysis. 
Differentiating Equations (41) and (6c) twice with respect to $\mathrm{x}$ and combining the results with Equation (41) yields

$$
\left(1+\frac{N}{\kappa G A}\right) \frac{d^{4} w(x)}{d x^{4}}-\left(\frac{N}{E I}+\frac{k}{\kappa G A}\right) \frac{d^{2} w(x)}{d x^{2}}+\frac{k}{E I} w(x)=\frac{q(x)}{E I}-\frac{1}{\kappa G A} \frac{d^{2} q(x)}{d x^{2}}
$$

Combining Equations (41) and (6c) yields

$$
\frac{M(x)}{E I}=-\left(1+\frac{N}{\kappa G A}\right) \frac{d^{2} w(x)}{d x^{2}}+\frac{k}{\kappa G A} w(x)-\frac{q(x)}{\kappa G A}
$$

The parameter $\mathrm{k}_{\mathrm{w}}$ of the Winkler foundation is defined in Equation (12b). Let us introduce the parameter $\mathrm{k}_{\mathrm{N}}$, as follows:

$$
N=k_{N} \frac{E I_{r}}{l^{2}}
$$

Substituting Equations (8c), (8e), (9a-e), (12b), and (42c) into the governing Equation (42a) yields

$$
\begin{aligned}
& \left(1+\frac{k_{N} \alpha_{r}}{\beta_{V k}}\right)\left(W_{i-2}-4 W_{i-1}+6 W_{i}-4 W_{i+1}+W_{i+2}\right)-\beta_{l k}^{2}\left(\frac{k_{N}}{\beta_{M k}}+\frac{k_{W} \alpha_{r}}{\beta_{V k}}\right) \frac{-W_{i-2}+16 W_{i-1}-30 W_{i}+16 W_{i+1}-W_{i+2}}{12} \\
& +\frac{k_{W} \beta_{l k}^{4}}{\beta_{M k}} W_{i}=\frac{q_{i} h^{4}}{\beta_{M k}}-\left.\frac{\alpha_{r}}{\beta_{V k} \beta_{l k}^{2}} h_{k}^{6} \frac{d^{2} q(x)}{d x^{2}}\right|_{i}
\end{aligned}
$$

The bending moments, the transverse forces, and the rotations of the cross sections are determined using Equations (42b) and (8e), Equations (37), (1b), (42b), (8d), and (8f), and Equations (4), (42b), (1b), (8d), and (8f), respectively.

$$
\begin{aligned}
M_{i}= & -\beta_{M k}\left(1+\frac{k_{N} \alpha_{r}}{\beta_{V k}}\right) \frac{-W_{i-2}+16 W_{i-1}-30 W_{i}+16 W_{i+1}-W_{i+2}}{12 h_{k}^{2}} \\
& +\frac{k_{W} \beta_{M k} \alpha_{r} \beta_{l k}^{2}}{\beta_{V k}} \frac{W_{i}}{h_{k}^{2}}-\frac{\beta_{M k} \alpha_{r}}{\beta_{V k}} l^{2} q_{i} \\
T_{i}=- & \beta_{M k}\left(1+\frac{k_{N} \alpha_{r}}{\beta_{V k}}\right) \frac{-W_{i-2}+2 W_{i-1}-2 W_{i+1}+W_{i+2}}{2 h_{k}^{3}} \\
& +\beta_{l k}^{2}\left(\frac{k_{W} \beta_{M k} \alpha_{r}}{\beta_{V k}}+k_{N}\right) \frac{W_{i-2}-8 W_{i-1}+8 W_{i+1}-W_{i+2}}{12 h_{k}^{3}}-\left.\frac{\beta_{M k} \alpha_{r}}{\beta_{V k}} l^{2} \frac{d q(x)}{d x}\right|_{i} \\
E I_{r} \varphi_{i}= & \frac{\beta_{M k} \alpha_{r}\left(1+\frac{k_{N} \alpha_{r}}{\beta_{V k}}\right) \frac{-W_{i-2}+2 W_{i-1}-2 W_{i+1}+W_{i+2}}{2 h_{k}}}{\beta_{V k}}\left(1-\frac{k_{W} \beta_{M k} \alpha_{r}^{2}}{\beta_{V K}^{2}}\right) \frac{W_{i-2}-8 W_{i-1}+8 W_{i+1}-W_{i+2}}{12 h_{k}}+\left.\frac{\beta_{M k} \alpha_{r}^{2}}{\beta_{V K}^{2}} l^{4} \frac{d q(x)}{d x}\right|_{i} \\
& +(1
\end{aligned}
$$




\subsubsection{M-W FDA in second-order analysis of a uniform beam}

Substituting Equations (2), (36), and (37) into Equation (35a) yields

$$
\frac{d^{2} M(x)}{d x^{2}}+N(x) \frac{d^{2} w(x)}{d x^{2}}-n(x) \frac{d w(x)}{d x}-k w(x)=-q(x)
$$

Substituting Equations (8a-b) and (9d-e) into Equation (43e) yields

$$
\begin{aligned}
& h_{k}^{2} M_{i-1}-2 h_{k}^{2} M_{i}+h_{k}^{2} M_{i+1}+\left(\frac{N_{i} h_{k}^{2}}{E I_{r}}+\frac{n_{i} h_{k}^{3}}{2 E I_{r}}\right) W_{i-1}-\left(\frac{2 N_{i} h_{k}^{2}}{E I_{r}}+\beta_{l k}^{4} \frac{k_{i} l^{4}}{E I_{r}}\right) W_{i} \\
& +\left(\frac{N_{i} h_{k}^{2}}{E I_{r}}-\frac{n_{i} h_{k}^{3}}{2 E I_{r}}\right) W_{i+1}=-q_{i} h_{k}^{4}
\end{aligned}
$$

The governing equations (Equations (43f) and (13e)) are applied at any point on the grid. The rotation of the cross section is calculated using Equations (13g). Applying Equations (37), (1b), and (8b) yields the transverse force, as follows:

$$
2 h_{k}^{3} T_{i}=h_{k}^{2} M_{i+1}-h_{k}^{2} M_{i-1}+\frac{N_{i} h_{k}^{2}}{E I_{r}}\left(W_{i+1}-W_{i-1}\right)
$$

\subsubsection{M FDA in second-order analysis of a uniform beam resting on Winkler foundation}

Fogang [6] derived following equations for the beam subjected to an axial effort and resting on an elastic Winkler foundation; the axial effort and the stiffness of the Winkler foundation were assumed constant in the beam.

$$
\begin{aligned}
& \left(1+\frac{N}{\kappa G A}\right) \frac{d^{4} M(x)}{d x^{4}}-\left(\frac{N}{E I}+\frac{k}{\kappa G A}\right) \frac{d^{2} M(x)}{d x^{2}}+\frac{k}{E I} M(x)=-\frac{d^{2} q(x)}{d x^{2}} \\
& k \times w(x)=\left(1+\frac{N}{\kappa G A}\right) \frac{d^{2} M(x)}{d x^{2}}-\frac{N}{E I} M(x)+q(x)
\end{aligned}
$$

Differentiating Equation (43i) with respect to $x$ and combining the result with Equations (1b), (4), and (37) yields the rotation angle and the transverse force as follows:

$$
\begin{aligned}
& k \times \varphi(x)=\left(1+\frac{N}{\kappa G A}\right) \frac{d^{3} M(x)}{d x^{3}}-\left(\frac{N}{E I}+\frac{k}{\kappa G A}\right) \frac{d M(x)}{d x}+\frac{d q(x)}{d x} \\
& T(x)=\frac{N}{k}\left(1+\frac{N}{\kappa G A}\right) \frac{d^{3} M(x)}{d x^{3}}+\left(1-\frac{N^{2}}{k E I}\right) \frac{d M(x)}{d x}+\frac{N}{k} \frac{d q(x)}{d x}
\end{aligned}
$$


Substituting Equations (8c), (8e), (9a-e), (12b), and (42c) into the governing Equation (43h) yields

$$
\begin{aligned}
& \left(1+\frac{k_{N} \alpha_{r}}{\beta_{V k}}\right)\left(M_{i-2}-4 M_{i-1}+6 M_{i}-4 M_{i+1}+M_{i+2}\right)-\beta_{l k}^{2}\left(\frac{k_{N}}{\beta_{M k}}+\frac{k_{W} \alpha_{r}}{\beta_{V k}}\right) \frac{-M_{i-2}+16 M_{i-1}-30 M_{i}+16 M_{i+1}-M_{i+2}}{12} \\
& +\frac{k_{W} \beta_{l k}^{4}}{\beta_{M k}} M_{i}=\left.h_{k}^{4} \frac{d^{2} q(x)}{d x^{2}}\right|_{i}
\end{aligned}
$$

Equation (431) is applied at any point on the grid. The deflections, the transverse forces, and the rotation angles are determined using Equations (43i), (8e), (9a-d), and (42c), Equations (43k), (8d), (8f), (9a-d), (12b), and (42c), and Equations (43j), (8d), (8f), (9a-d), (12b), and (42c), respectively.

$$
\begin{aligned}
k \times w_{i}= & \left(1+\frac{k_{N} \alpha_{r}}{\beta_{V k}}\right) \frac{-M_{i-2}+16 M_{i-1}-30 M_{i}+16 M_{i+1}-M_{i+2}}{12 h_{k}^{2}}-\frac{k_{N}}{\beta_{M k} l^{2}} M_{i}+q_{i} \\
k \times \varphi_{i}= & \left(1+\frac{k_{N} \alpha_{r}}{\beta_{V k}}\right) \frac{-M_{i-2}+2 M_{i-1}-2 M_{i+1}+M_{i+2}}{2 h_{k}^{3}} \\
& -\beta_{l k}^{2}\left(\frac{k_{N}}{\beta_{M k}}+\frac{k_{W} \alpha_{r}}{\beta_{V k}}\right) \frac{M_{i-2}-8 M_{i-1}+8 M_{i+1}-M_{i+2}}{12 h_{k}^{3}}+\left.\frac{d q(x)}{d x}\right|_{i} \\
T_{i}=\frac{k_{N}}{k_{W} \beta_{l k}^{2}} & \left(1+\frac{k_{N} \alpha_{r}}{\beta_{V k}}\right) \frac{-M_{i-2}+2 M_{i-1}-2 M_{i+1}+M_{i+2}}{2 h_{k}} \\
& +\left(1-\frac{k_{N}^{2}}{k_{W} \beta_{M k}}\right) \frac{M_{i-2}-8 M_{i-1}+8 M_{i+1}-M_{i+2}}{12 h_{k}}+\left.\frac{k_{N} l^{2}}{k_{W}} \frac{d q(x)}{d x}\right|_{i}
\end{aligned}
$$

\subsubsection{Second-order analysis of a tapered beam}

\subsubsection{1}

W- $\Phi$ FDA in second-order analysis of a tapered beam

Substituting Equations (4), (36), and (37) into Equation (35a) yields

$$
\begin{aligned}
& \frac{d \kappa G A(x)}{d x} \times\left(\frac{d w(x)}{d x}-\varphi(x)\right)+\kappa G A(x) \times\left(\frac{d^{2} w(x)}{d x^{2}}-\frac{d \varphi(x)}{d x}\right) \\
& +N(x) \frac{d^{2} w(x)}{d x^{2}}-n(x) \frac{d w(x)}{d x}-k w(x)=-q(x)
\end{aligned}
$$




\section{TIMOSHENKO BEAM THEORY USING THE FINITE DIFFERENCE METHOD}

The grid spacing in segment $\mathrm{k}$ is $\mathrm{h}_{\mathrm{k}}$. The reference flexural rigidity $\mathrm{EI}_{\mathrm{r}}$, the reference shear stiffness $\kappa G \mathrm{~A}_{\mathrm{r}}$, and the parameters $\alpha_{\mathrm{r}}, \beta_{\mathrm{Vi}}$, and $\beta_{\mathrm{Vi}}^{\prime}$ (Equations (9c-f) and (14a-d)) are defined. Substituting Equations (8a-b), (9c-f), and (14a-d) into Equation (44a) yields

$$
\begin{aligned}
& \left(-\frac{\beta_{V i}^{\prime} \beta_{l k}^{2}}{2 \alpha_{r}}+\frac{\beta_{V i} \beta_{l k}^{2}}{\alpha_{r}}+\frac{N_{i} h_{k}^{2}}{E I_{r}}+\frac{n_{i} h_{k}^{3}}{2 E I_{r}}\right) W_{i-1}-\left(\frac{2 \beta_{V i} \beta_{l k}^{2}}{\alpha_{r}}+\frac{2 N_{i} h_{k}^{2}}{E I_{r}}+\beta_{l k}^{4} \frac{k_{i} l^{4}}{E I_{r}}\right) W_{i} \\
& +\left(\frac{\beta_{V i}^{\prime} \beta_{l k}^{2}}{2 \alpha_{r}}+\frac{\beta_{V i} \beta_{l k}^{2}}{\alpha_{r}}+\frac{N_{i} h_{k}^{2}}{E I_{r}}-\frac{n_{i} h_{k}^{3}}{2 E I_{r}}\right) W_{i+1}+\frac{\beta_{V i} \beta_{l k}^{2}}{2 \alpha_{r}} h_{k} \Phi_{i-1}-\frac{\beta_{V i}^{\prime} \beta_{l k}^{2}}{\alpha_{r}} h_{k} \Phi_{i}-\frac{\beta_{V i} \beta_{l k}^{2}}{2 \alpha_{r}} h_{k} \Phi_{i+1}=-q_{i} h_{k}^{4}
\end{aligned}
$$

Equations (15b) and (44b) are applied at any point on the grid. The bending moments and the transverse forces are calculated using Equations (11a) and (40), respectively, $\beta_{\mathrm{Mk}}$ and $\beta_{\mathrm{Vk}}$ being replaced by $\beta_{\mathrm{Mi}}$ and $\beta_{\mathrm{Vi}}$.

\subsubsection{M-W FDA in second-order analysis of a tapered beam}

The governing equations (Equations (16) and (43f)) are applied at any point on the grid. The rotations of the cross sections and the transverse forces are calculated using Equations (13g) and (43g), respectively. However, $\beta_{\mathrm{Vk}}$ is replaced by $\beta_{\mathrm{Vi}}$ in Equation (13g).

\subsubsection{Second-order element stiffness matrix of a uniform beam}

The beam is divided in $\mathrm{n}$ parts of equal length $\mathrm{h}_{\mathrm{k}}$, as shown in Figure 10.
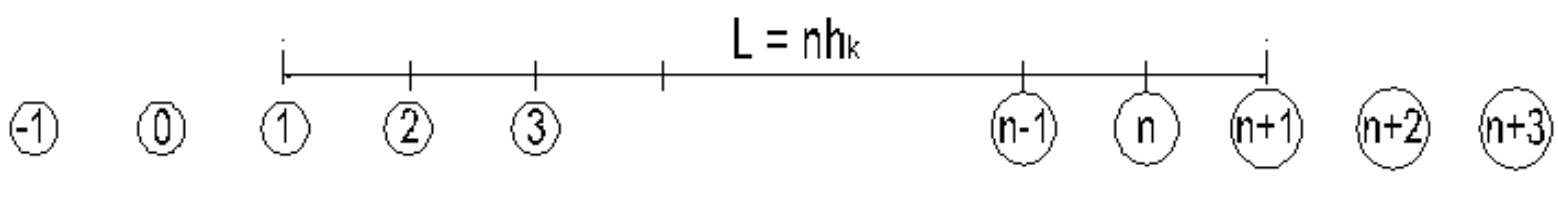

Figure 10 FDM discretization for $4 \times 4$ element stiffness matrix

The sign convention for bending moments, transverse forces, displacements, and rotations of the cross sections adopted to determine the element stiffness matrix in local coordinates is illustrated in Figure 7, the shear forces $V_{i}$ and $V_{k}$ being replaced by the transverse forces $T_{i}$ and $T_{k}$. The W FDA is applied here (Details in Appendix D). The W- $\Phi$ FDA and M-W FDA (Details in Appendix D) can also be considered using appropriate formulas developed in previous sections. Equation (43a) with $\mathrm{qi}=0$ and $\mathrm{k}_{\mathrm{W}}=0$ is applied at any point on the grid (nodes $1,2, \ldots \mathrm{n}+1$ of Figure 10).

The static compatibility boundary conditions are applied using Equations (43b-c). The geometric compatibility boundary conditions are applied similarly to Equations (26a-d), the rotation of the cross section being formulated with Equation (43d). The analysis continues similarly to Section 2.1.4.1.1 (Equations (27)-(30b)). 


\section{TIMOSHENKO BEAM THEORY USING THE FINITE DIFFERENCE METHOD}

\subsubsection{Second-order element stiffness matrix of a tapered beam}

The M-W FDM approximation is applied here. The W- $\Phi$ FDM approximation can also be applied with appropriate formulas developed previously. The sign convention for bending moments, transverse forces, displacements, and rotations of the cross sections adopted to determine the element stiffness matrix in local coordinates is illustrated in Figure 7, the shear forces $V_{i}$ and $V_{k}$ being replaced by the transverse forces $T_{i}$ and $T_{k}$.

Equations (16) and (43f) with $\mathrm{qi}=0$ and $\mathrm{ki}=0$ are applied at any point on the grid (nodes $1,2, \ldots \mathrm{n}+1$ of Figure 8). The static compatibility boundary conditions are expressed similarly to Equations (31a-d); however, the shear forces are replaced by the transverse forces (Equation $(43 \mathrm{~g})$ ). The geometric compatibility boundary conditions are the same as in Section 2.1.4.1.2 (Equations (31e-h)). The analysis continues similarly to Section 2.1.4.1.1 (Equations (27)-(30b)).

\subsection{Vibration analysis of the Timoshenko beam}

\subsubsection{Free vibration analysis}

It is focused here on the natural frequencies of the beam. A second-order analysis is conducted; and the first-order analysis can easily be deduced. The equations of dynamic equilibrium on an infinitesimal beam element are as follows:

$$
\begin{aligned}
& \frac{\partial T^{*}(x, t)}{\partial x}-k(x) w^{*}(x, t)=\rho A(x) \frac{\partial^{2} w^{*}(x, t)}{\partial t^{2}} \\
& \frac{\partial M^{*}(x, t)}{\partial x}+N(x) \frac{\partial w^{*}(x, t)}{\partial x}-T^{*}(x, t)=-\rho I(x) \frac{\partial^{2} \varphi^{*}(x, t)}{\partial t^{2}}
\end{aligned}
$$

where $\rho$ is the beam's mass per unit volume, $\mathrm{A}(x)$ is the cross-sectional area, $\mathrm{N}(x)$ is the axial force (positive in tension), and $\mathrm{k}(x)$ is the stiffness of the elastic Winkler foundation. A harmonic vibration being assumed, $\mathrm{T}^{*}(x, t), \mathrm{M}^{*}(x, t), \mathrm{w}^{*}(x, t)$, and $\varphi^{*}(x, t)$ can be expressed as follows $\left(\mathrm{S}^{*}(x, t)\right.$ representing $\mathrm{T}^{*}(x, t), \mathrm{M}^{*}(x, t), \mathrm{w}^{*}(x, t)$, and $\left.\varphi^{*}(x, t)\right)$ :

$$
S^{*}(x, t)=S(x) \times \sin (\omega t+\theta)
$$

where $\omega$ is the circular frequency of the beam. Substituting Equation (47) into Equations (45) and (46) yields

$$
\begin{aligned}
& \frac{d T(x)}{d x}-k(x) w(x)+\rho A(x) \omega^{2} w(x)=0 \\
& \frac{d M(x)}{d x}+N(x) \frac{d w(x)}{d x}-T(x)-\rho I(x) \omega^{2} \varphi(x)=0
\end{aligned}
$$

Substituting Equations (36) and (37) into Equations (48a-b) yields

$$
\begin{aligned}
& \frac{d V(x)}{d x}+N(x) \frac{d^{2} w(x)}{d x^{2}}-n(x) \frac{d w(x)}{d x}-k(x) w(x)+\rho A(x) \omega^{2} w(x)=0 \\
& \frac{d M(x)}{d x}-V(x)-\rho I(x) \omega^{2} \varphi(x)=0
\end{aligned}
$$




\subsubsection{Free vibration analysis of uniform beams within segments}

\subsubsection{1 $\quad W-\Phi$ FDA in free vibration analysis of a uniform beam}

A reference flexural stiffness $\mathrm{EI}_{\mathrm{r}}$, a reference shear stiffness $\kappa \mathrm{GA}$, parameters $\beta_{\mathrm{Mk}}, \beta_{\mathrm{Vk}}$, and $\alpha_{\mathrm{r}}$ were defined in Equations (9a-c). A reference cross-sectional area $A_{r}$ and a reference length $l_{r}$ are defined and related to the crosssectional area $A_{k}$ and the grid spacing $h_{k}$ in the segment, as follows:

$$
A_{k}=\beta_{A K} A_{r} \text { (50a) } \quad h_{k}=\beta_{l k} l_{r}
$$

The parameter $\alpha_{r}$ (Equation (9c)) is defined with $1_{r}$ instead of 1 . Substituting Equations (3) and (4) into Equations (49ab) and combining the results with Equations (8a-b) yields the following FDM formulations:

$$
\begin{aligned}
& \left(\frac{\beta_{V k} \beta_{l k}^{2}}{\alpha_{r}}+\frac{N_{i} h_{k}^{2}}{E I_{r}}+\frac{n_{i} h_{k}^{3}}{2 E I_{r}}\right) W_{i-1}-\left(2 \frac{\beta_{V k} \beta_{l k}^{2}}{\alpha_{r}}+\frac{2 N_{i} h_{k}^{2}}{E I_{r}}+\beta_{l k}^{4} \frac{k_{i} l_{r}^{4}}{E I_{r}}-\frac{\beta_{A k} \beta_{l k}^{4} \rho A_{r} \omega^{2} l_{r}^{4}}{E I_{r}}\right) W_{i} \\
& +\left(\frac{\beta_{V k} \beta_{l k}^{2}}{\alpha_{r}}+\frac{N_{i} h_{k}^{2}}{E I_{r}}-\frac{n_{i} h_{k}^{3}}{2 E I_{r}}\right) W_{i+1}+\frac{\beta_{V k} \beta_{l k}^{2}}{2 \alpha_{r}} h_{k} \Phi_{i-1}-\frac{\beta_{V k} \beta_{l k}^{2}}{2 \alpha_{r}} h_{k} \Phi_{i+1}=0 \\
& -\frac{\beta_{V k} \beta_{l k}^{2}}{2 \alpha_{r}} W_{i-1}+\frac{\beta_{V k} \beta_{l k}^{2}}{2 \alpha_{r}} W_{i+1}+\beta_{M k} h_{k} \Phi_{i-1}-\left(2 \beta_{M k}+\frac{\beta_{V k} \beta_{l k}^{2}}{\alpha_{r}}-\frac{\rho I_{k} \omega^{2} h_{k}^{2}}{E I_{r}}\right) h_{k} \Phi_{i}+\beta_{M k} h_{k} \Phi_{i+1}=0
\end{aligned}
$$

The reference coefficient of rotary inertia $\mathrm{k}_{\mathrm{RIr}}$ and the vibration frequency $\omega$ are defined as follows

$$
k_{R I r}=\frac{I_{r}}{A_{r} l_{r}^{2}}
$$

$$
\omega=\lambda \times \sqrt{\frac{E I_{r}}{\rho A_{r} l_{r}^{4}}}
$$

Substituting Equations (52a-b) into Equations (51a-b) yields

$$
\begin{aligned}
& \left(\frac{\beta_{V k} \beta_{l k}^{2}}{\alpha_{r}}+\frac{N_{i} h_{k}^{2}}{E I_{r}}+\frac{n_{i} h_{k}^{3}}{2 E I_{r}}\right) W_{i-1}-\left(\frac{2 \beta_{V k} \beta_{l k}^{2}}{\alpha_{r}}+\frac{2 N_{i} h_{k}^{2}}{E I_{r}}+\beta_{l k}^{4} \frac{k_{i} l_{r}^{4}}{E I_{r}}-\beta_{A k} \beta_{l k}^{4} \lambda^{2}\right) W_{i} \\
& +\left(\frac{\beta_{V k} \beta_{l k}^{2}}{\alpha_{r}}+\frac{N_{i} h_{k}^{2}}{E I_{r}}-\frac{n_{i} h_{k}^{3}}{2 E I_{r}}\right) W_{i+1}+\frac{\beta_{V k} \beta_{l k}^{2}}{2 \alpha_{r}} h_{k} \Phi_{i-1}-\frac{\beta_{V k} \beta_{l k}^{2}}{2 \alpha_{r}} h_{k} \Phi_{i+1}=0 \\
& -\frac{\beta_{V k} \beta_{l k}^{2}}{2 \alpha_{r}} W_{i-1}+\frac{\beta_{V k} \beta_{l k}^{2}}{2 \alpha_{r}} W_{i+1}+\beta_{M k} h_{k} \Phi_{i-1}-\left(2 \beta_{M k}+\frac{\beta_{V k} \beta_{l k}^{2}}{\alpha_{r}}-k_{R I r} \beta_{M k} \beta_{l k}^{2} \lambda^{2}\right) h_{k} \Phi_{i} \\
& +\beta_{M k} h_{k} \Phi_{i+1}=0
\end{aligned}
$$




\section{TIMOSHENKO BEAM THEORY USING THE FINITE DIFFERENCE METHOD}

Equations (53a-b) are applied at any point on the grid. The bending moments and transverse forces are determined using Equations (11a) and (40), respectively.

For the special case of a uniform beam without an axial force or a Winkler foundation, Equations (53a-b) become

$$
\begin{aligned}
& W_{i-1}-\left(2-\alpha_{r} \beta_{l k}^{2} \lambda^{2}\right) W_{i}+W_{i+1}+\frac{1}{2} h_{k} \Phi_{i-1}-\frac{1}{2} h_{k} \Phi_{i+1}=0 \\
& -\frac{\beta_{l k}^{2}}{2 \alpha_{r}} W_{i-1}+\frac{\beta_{l k}^{2}}{2 \alpha_{r}} W_{i+1}+h_{k} \Phi_{i-1}-\left(2+\frac{\beta_{l k}^{2}}{\alpha_{r}}-k_{R I r} \beta_{l k}^{2} \lambda^{2}\right) h_{k} \Phi_{i}+h_{k} \Phi_{i+1}=0
\end{aligned}
$$

\section{Effect of a concentrated mass, or a spring}

The dynamic behavior of a beam carrying a concentrated mass or having a spring was analyzed, as shown in Figure 11.

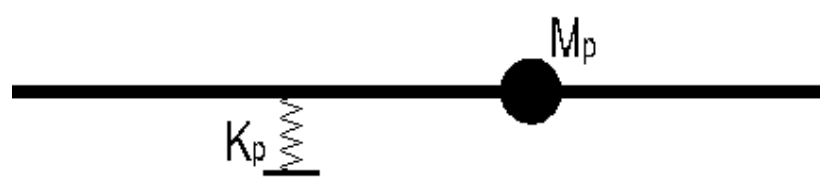

Figure 11 Vibration of beam having a concentrated mass and a spring

The stiffness of the spring is $K_{p}$, and the concentrated mass is $M_{p}$.

$$
\begin{aligned}
& K_{p}=k_{p} \times E I_{r} / l_{r}^{3} \\
& M_{p}=m_{p} \times \rho A_{r} l_{r}
\end{aligned}
$$

The continuity equations for deflections, rotations of the cross sections, and bending moments are defined in Equations (20a), (20b), and (20c), respectively. Equation (20c) is applied with $\mathrm{M}^{*}=0$. The reference length of the beam is $1_{\mathrm{r}}$ (Equation (50b)).

Applying Equations (9e), (50b), (52b), and (55a-b), the balance of vertical forces in the case of a concentrated mass or a spring yields

$$
\begin{aligned}
& T_{i l}-T_{i r}-\frac{M_{p} \omega^{2}}{E I_{r}} W_{i l}=0 \rightarrow T_{i l}-T_{i r}-\frac{m_{p}}{l_{r}^{3}} \lambda^{2} W_{i l}=0 \\
& T_{i l}-T_{i r}+\frac{K_{p}}{E I_{r}} W_{i l}=0 \rightarrow T_{i l}-T_{i r}+\frac{k_{p}}{l_{r}^{3}} W_{i l}=0,
\end{aligned}
$$

respectively. The transverse forces $\mathrm{Til}$ and $\mathrm{Tir}$ are calculated using Equation (40).

Effect of a spring-mass system: The dynamic behavior of a beam carrying a spring-mass system was analyzed, as represented in Figure 12. The deflection of the mass is denoted by $\mathrm{W}_{\mathrm{iM}}$. 


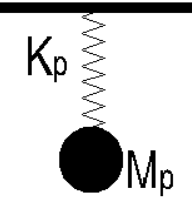

Figure 12 Vibration of a beam carrying a spring-mass system

Applying Equations (9e), (50b), (52b), and (55a-b), the balance of vertical forces yields

$$
\begin{aligned}
& T_{i l}-T_{i r}-\frac{M_{p} \omega^{2}}{E I_{r}} W_{i M}=0 \rightarrow T_{i l}-T_{i r}-\frac{m_{p}}{l_{r}^{3}} \lambda^{2} W_{i M}=0 \\
& \frac{M_{p} \omega^{2}}{E I_{r}} W_{i M}=\frac{K_{p}}{E I_{r}} \times\left(W_{i M}-W_{i r}\right) \rightarrow m_{p} \lambda^{2} W_{i M}=k_{p}\left(W_{i M}-W_{i r}\right)
\end{aligned}
$$

\subsection{M-W FDA in free vibration analysis of a uniform beam}

Substituting Equation (49b) into Equation (49a) and combining the result with Equation (3) yields

$$
\frac{d^{2} M(x)}{d x^{2}}+\frac{\rho \omega^{2}}{E} M(x)+N(x) \frac{d^{2} w(x)}{d x^{2}}-n(x) \frac{d w(x)}{d x}-k(x) w(x)+\rho A \omega^{2} w(x)=0
$$

Fogang [11] presented the following relationship that combines bending, shear, curvature, and natural frequency:

$$
\frac{d^{2} w(x)}{d x^{2}}+\left(1-\frac{\rho I \omega^{2}}{\kappa G A}\right) \times \frac{M(x)}{E I}-\frac{1}{\kappa G A} \times \frac{d^{2} M(x)}{d x^{2}}=0
$$

In [11] the relationship between shear force/rotation of the cross section and bending moment/deflection is presented as follows:

$$
\begin{aligned}
& \left(1-\frac{\rho I \omega^{2}}{\kappa G A}\right) \times V(x)=\frac{d M(x)}{d x}-\rho I \omega^{2} \frac{d w(x)}{d x} \\
& \left(\rho I \omega^{2}-\kappa G A\right) \times \varphi(x)=\frac{d M(x)}{d x}-\kappa G A \frac{d w(x)}{d x}
\end{aligned}
$$

Substituting Equations (8a-b), (50a-b), and (52a-b) into Equations (58) and (59a) yields

$$
\begin{aligned}
& h_{k}^{2} M_{i-1}+\left(k_{R I r} \beta_{l k}^{2} \lambda^{2}-2\right) h_{k}^{2} M_{i}+h_{k}^{2} M_{i+1}+\left(\frac{N_{i} h_{k}^{2}}{E I_{r}}+\frac{n_{i} h_{k}^{3}}{2 E I_{r}}\right) W_{i-1} \\
& -\left(\frac{2 N_{i} h_{k}^{2}}{E I_{r}}+\beta_{l k}^{4} \frac{k_{i} l_{r}^{4}}{E I_{r}}-\beta_{A k} \beta_{l k}^{4} \lambda^{2}\right) W_{i}+\left(\frac{N_{i} h_{k}^{2}}{E I_{r}}-\frac{n_{i} h_{k}^{3}}{2 E I_{r}}\right) W_{i+1}=0
\end{aligned}
$$


TIMOSHENKO BEAM THEORY USING THE FINITE DIFFERENCE METHOD

$W_{i-1}-2 W_{i}+W_{i+1}-\frac{\alpha_{r}}{\beta_{V k} \beta_{l k}^{2}} h_{k}^{2} M_{i-1}+\left(\frac{1}{\beta_{M k}}+\frac{2 \alpha_{r}}{\beta_{V k} \beta_{l k}^{2}}-\frac{1}{\beta_{V k}} k_{R I r} \alpha_{r} \lambda^{2}\right) h_{k}^{2} M_{i}$

$-\frac{\alpha_{r}}{\beta_{V k} \beta_{l k}^{2}} h_{k}^{2} M_{i+1}=0$

Equations (60a-b) are applied at any point on the grid. Substituting Equations (8a-b), (9c), and (52a-b) into Equations (59b-c) yields the shear force and the rotation of the cross section, as follows:

$$
\begin{aligned}
& \left(1-\frac{\beta_{M k}}{\beta_{V k}} k_{R I r} \alpha_{r} \lambda^{2}\right) V_{i}=\frac{M_{i+1}-M_{i-1}}{2 h_{k}}-\beta_{M k} \beta_{l k}^{2} k_{R I r} \lambda^{2} \frac{W_{i+1}-W_{i-1}}{2 h_{k}^{3}} \\
& \left(\beta_{M k} \beta_{l k}^{2} k_{R I r} \lambda^{2}-\frac{\beta_{V k} \beta_{l k}^{2}}{\alpha_{r}}\right) \frac{E I_{r}}{h_{k}^{2}} \varphi_{i}=\frac{M_{i+1}-M_{i-1}}{2 h_{k}}-\frac{\beta_{V k} \beta_{l k}^{2}}{\alpha_{r}} \frac{W_{i+1}-W_{i-1}}{2 h_{k}^{3}}
\end{aligned}
$$

The transverse force is calculated using Equations (37) and (60c).

The dynamic behavior of a beam carrying a concentrated mass, a spring, or a spring-mass system was analyzed similarly to the previous section (Equations (55a)-(57b)).

\subsubsection{Free vibration analysis of tapered beams}

The W- $\Phi$ FDM approximation was considered for the vibration analysis of tapered beams. The M-W FDM approximation led to complicated expressions and was not further analyzed. The beam segment with length 1 is divided in parts of equal length $h_{k}$. The reference values of flexural stiffness, shear stiffness, cross-sectional area, and coefficient of rotary inertia are defined like in previous sections.

Substituting Equations (3) and (4) into Equations (49a-b) and combining the results with Equations (8a-b), (14a-d), (50a-b), and (52a-b) yields the following FDM formulations of the governing equations:

$$
\begin{aligned}
& \left(-\frac{\beta_{V i}^{\prime} \beta_{l k}^{2}}{2 \alpha_{r}}+\frac{\beta_{V i} \beta_{l k}^{2}}{\alpha_{r}}+\frac{N_{i} h_{k}^{2}}{E I_{r}}+\frac{n_{i} h_{k}^{3}}{2 E I_{r}}\right) W_{i-1}-\left(\frac{2 \beta_{V i} \beta_{l k}^{2}}{\alpha_{r}}+\frac{2 N_{i} h_{k}^{2}}{E I_{r}}+\beta_{l k}^{4} \frac{k_{i} l_{r}^{4}}{E I_{r}}-\beta_{A i} \beta_{l k}^{4} \lambda^{2}\right) W_{i} \\
& +\left(\frac{\beta_{V i}^{\prime} \beta_{l k}^{2}}{2 \alpha_{r}}+\frac{\beta_{V i} \beta_{l k}^{2}}{\alpha_{r}}+\frac{N_{i} h_{k}^{2}}{E I_{r}}-\frac{n_{i} h_{k}^{3}}{2 E I_{r}}\right) W_{i+1}+\frac{\beta_{V i} \beta_{l k}^{2}}{2 \alpha_{r}} h_{k} \Phi_{i-1}-\frac{\beta_{V i}^{\prime} \beta_{l k}^{2}}{\alpha_{r}} h_{k} \Phi_{i}-\frac{\beta_{V i} \beta_{l k}^{2}}{2 \alpha_{r}} h_{k} \Phi_{i+1}=0
\end{aligned}
$$




$$
\begin{aligned}
& -\frac{\beta_{V i} \beta_{l k}^{2}}{2 \alpha_{r}} W_{i-1}+\frac{\beta_{V i} \beta_{l k}^{2}}{2 \alpha_{r}} W_{i+1}+\left(\beta_{M i}-\frac{\beta_{M i}^{\prime}}{2}\right) h_{k} \Phi_{i-1}-\left(2 \beta_{M i}+\frac{\beta_{V i} \beta_{l k}^{2}}{\alpha_{r}}-k_{R I r} \beta_{M i} \beta_{l k}^{2} \lambda^{2}\right) h_{k} \Phi_{i} \\
& +\left(\beta_{M i}+\frac{\beta_{M i}^{\prime}}{2}\right) h_{k} \Phi_{i+1}=0
\end{aligned}
$$

Equations (61a-b) are applied at any point on the grid. The bending moments and transverse forces are determined using Equations (11a) and (40), respectively, $\beta_{\mathrm{Mk}}$ and $\beta_{\mathrm{Vk}}$ being replaced by $\beta_{\mathrm{Mi}}$ and $\beta_{\mathrm{Vi}}$.

\section{Effect of a concentrated mass, a spring, or a spring-mass system}

The dynamic behavior of a beam carrying a concentrated mass, a spring, or a spring-mass system is analyzed similarly to the previous section (Equations (55a)-(57b). The transverse forces Til and Tir are calculated using Equation (40), $\beta_{\mathrm{Vk}}$ being replaced by $\beta_{\mathrm{Vi}}$.

\subsubsection{Direct time integration method}

The direct time integration method (DTIM) developed here describes the dynamic response of a beam as a multi-degreeof-freedom system. Viscosity $\eta$ and external loading $\mathrm{p}(x, t)$ are considered.

\subsubsection{DTIM for uniform beams within segments}

The W-Ф FDA was considered for the vibration analysis of the uniform beam. Substituting Equations (3), (4), (36), and (37) into Equations (45) and (46) yields the following governing equation:

$$
\begin{aligned}
& \kappa G A \times\left(\frac{\partial^{2} w^{*}(x, t)}{\partial x^{2}}-\frac{\partial \varphi^{*}(x, t)}{\partial x}\right)-n(x) \frac{\partial w^{*}(x, t)}{\partial x}+N(x) \frac{\partial^{2} w^{*}(x, t)}{\partial x^{2}} \\
& -k(x) w^{*}(x, t)-\rho A \frac{\partial^{2} w^{*}(x, t)}{\partial t^{2}}-\eta \frac{\partial w^{*}(x, t)}{\partial t}=-p(x, t) \\
& E I \frac{\partial^{2} \varphi^{*}(x, t)}{\partial x^{2}}+\kappa G A \times\left(\frac{\partial w^{*}(x, t)}{\partial x}-\varphi^{*}(x, t)\right)-\rho I \frac{\partial^{2} \varphi^{*}(x, t)}{\partial t^{2}}=0
\end{aligned}
$$

The derivatives with respect to $x$ are formulated using Equations (8a-b), while those with respect to $t$ (time increment is $\Delta \mathrm{t})$ are formulated considering a three-point stencil with Equations (63a-c):

$$
\left.\frac{\partial w^{*}(x, t)}{\partial t}\right|_{i, t}=\left.\frac{-w_{i, t-\Delta t}^{*}+w_{i, t+\Delta t}^{*}}{2 \Delta t} \quad \frac{\partial^{2} w^{*}(x, t)}{\partial t^{2}}\right|_{i, t}=\frac{w_{i, t-\Delta t}^{*}-2 w_{i, t}^{*}+w_{i, t+\Delta t}^{*}}{\Delta t^{2}}
$$


At initial time $\mathbf{t}=\mathbf{0}$, a three-point forward difference approximation is applied (Equation (18a)):

$$
\left.\frac{\partial^{2} w^{*}}{\partial t^{2}}\right|_{i, 0}=\left.\frac{w_{i, 0}^{*}-2 w_{i, \Delta t}^{*}+w_{i, 2 \Delta t}^{*}}{\Delta t^{2}} \quad \frac{\partial w^{*}}{\partial t}\right|_{i, 0}=\frac{-3 w_{i, 0}^{*}+4 w_{i, \Delta t}^{*}-w_{i, 2 \Delta t}^{*}}{2 \Delta t}
$$

At final time $\mathbf{t}=\mathbf{T}$, a three-point backward difference approximation is applied (Equation (18c)):

$$
\left.\frac{\partial^{2} w^{*}}{\partial t^{2}}\right|_{i, T}=\left.\frac{w_{i, T-2 \Delta t}^{*}-2 w_{i, T-\Delta t}^{*}+w_{i, T}^{*}}{\Delta t^{2}} \quad \frac{\partial w^{*}}{\partial t}\right|_{i, T}=\frac{w_{i, T-2 \Delta t}^{*}-4 w_{i, T-\Delta t}^{*}+3 w_{i, T}^{*}}{2 \Delta t}
$$

The governing equations (Equations (62a-b)) can be formulated with the FDM for $\mathrm{x}=\mathrm{i}$ at time $\mathrm{t}$. The FDM formulations of these equations are applied at any point of the beam at any time $t$ using a five-point stencil. Additional points are introduced to satisfy the boundary and continuity conditions. The boundary conditions are satisfied using a three-point stencil. Thus, beam deflection $\mathrm{w}^{*}(x, t)$ and rotation $\varphi^{*}(x, t)$ can be determined with the Cartesian model represented in Figure 13. The bending moment $\mathrm{M}^{*}(x, t)$, shear force $\mathrm{V}^{*}(x, t)$, and transverse force $\mathrm{T}^{*}(x, t)$ are calculated using Equations (11a-b) and (37), respectively.

With this model, the assumptions made previously can be verified, namely the separation of variables and the harmonic vibration (Equation (47)).

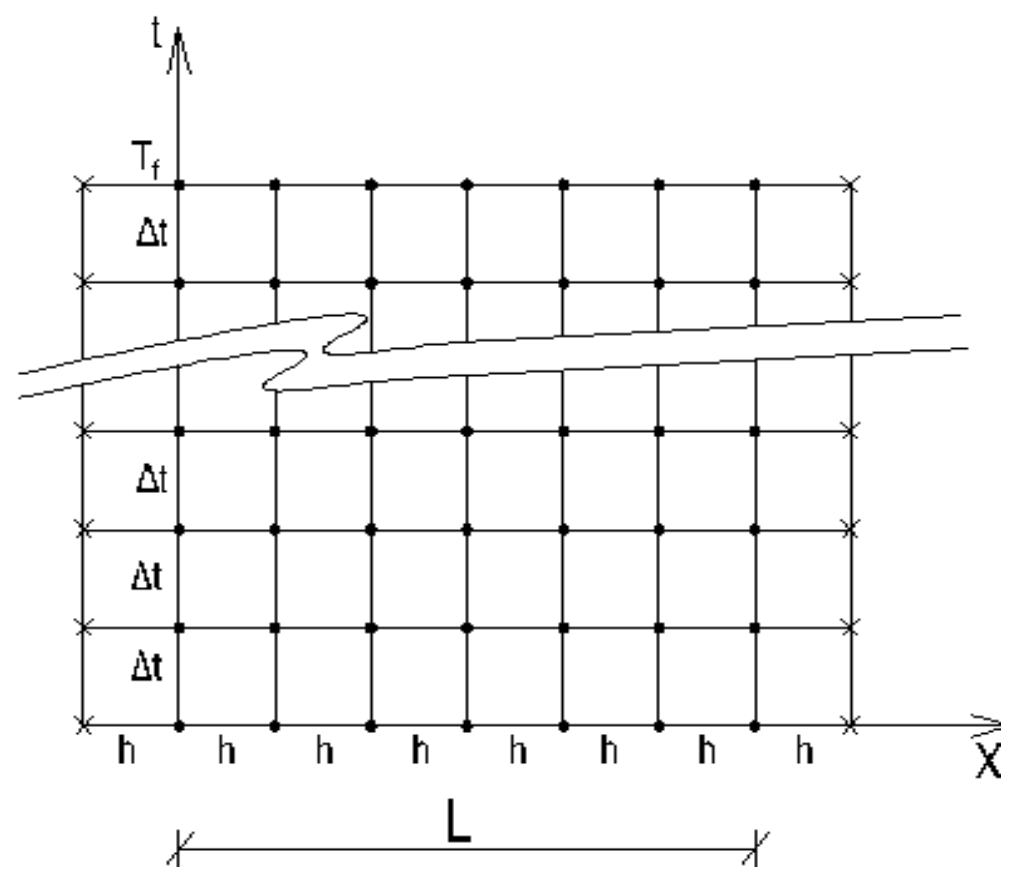

Figure 13 Model for the calculation of time-dependent vibration of a uniform beam 


\subsubsection{DTIM for tapered beams}

A similar analysis can be conducted. Thus, Equations (62a-b) become

$\frac{d \kappa G A(x)}{d x}\left(\frac{\partial w^{*}(x, t)}{\partial x}-\varphi^{*}(x, t)\right)+\kappa G A(x)\left(\frac{\partial^{2} w^{*}(x, t)}{\partial x^{2}}-\frac{\partial \varphi^{*}(x, t)}{\partial x}\right)$

$-n(x) \frac{\partial w^{*}(x, t)}{\partial x}+N(x) \frac{\partial^{2} w^{*}(x, t)}{\partial x^{2}}-k(x) w^{*}(x, t)-\rho A(x) \frac{\partial^{2} w^{*}(x, t)}{\partial t^{2}}-\eta \frac{\partial w^{*}(x, t)}{\partial t}=-p(x, t)$

$\frac{d E I(x)}{d x} \frac{\partial \varphi^{*}(x, t)}{\partial x}+E I(x) \frac{\partial^{2} \varphi^{*}(x, t)}{\partial x^{2}}+\kappa G A(x) \times\left(\frac{\partial w^{*}(x, t)}{\partial x}-\varphi^{*}(x, t)\right)-\rho I(x) \frac{\partial^{2} \varphi^{*}(x, t)}{\partial t^{2}}=0$

The derivatives with respect to $x$ are formulated using Equations (8a-b), while those with respect to $t$ (time increment is

$\Delta \mathrm{t}$ ) are formulated considering a three-point stencil with Equations (63a-c). The FDM formulations of Equations (64a-b)

are applied at any point on the beam and at any time t using a five-point stencil. Additional points are introduced to satisfy the boundary and continuity conditions. The boundary conditions are satisfied using a three-point stencil. Thus, beam deflection $\mathrm{w}^{*}(x, t)$ and rotation $\varphi^{*}(x, t)$ can be determined with the Cartesian model represented in Figure 13. The bending moment $\mathrm{M}^{*}(x, t)$, shear force $\mathrm{V}^{*}(x, t)$, and transverse force $\mathrm{T}^{*}(x, t)$ are calculated using Equations (11a-b) and (37), respectively, $\beta_{\mathrm{Vk}}$ being replaced by $\beta_{\mathrm{Vi}}$ in Equation (11b) and $\beta_{\mathrm{Mk}}$ by $\beta_{\mathrm{Mi}}$ in Equation (11a).

With this model, the assumptions made previously can be verified, namely the separation of variables and the harmonic vibration (Equation (47)).

\section{Results and discussion}

\subsection{First-order analysis}

\subsubsection{Beam subjected to a uniformly distributed load}

A uniform fixed-pinned beam subjected to a uniformly distributed load, as shown in Figure 14, was analyzed.

\section{$p=10.0 \mathrm{kN} / \mathrm{m}$}

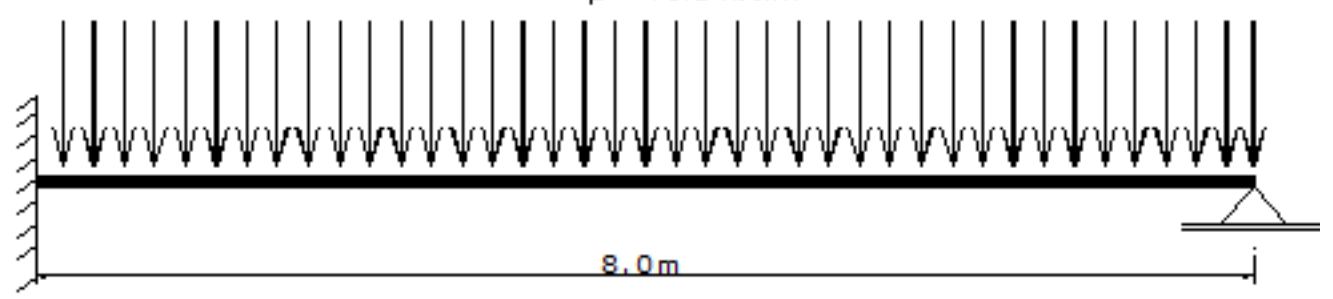

Figure 14 Uniform fixed-pinned beam subjected to a uniformly distributed load 


\section{TIMOSHENKO BEAM THEORY USING THE FINITE DIFFERENCE METHOD}

The governing equations (Equations (10a-b)) were applied at grid points 1, 2, 3, 4, and 5. The boundary conditions were satisfied using Equation (11a).

The bending shear factor $\alpha=\mathrm{EI} / \kappa \mathrm{GAl}{ }^{2}=0.025$

(Equation (9c))

The analysis was conducted with W- $-\Phi$ FDA, M-W FDA, and W FDA. Details of the analysis and results are presented in Appendix A and in the Supplementary Material "Fixed-pinned beam subjected to a uniformly distributed load." Table 1 lists the exact results obtained with classical beam theory (CBT) and those obtained with W- $\Phi$ FDA and M-W FDA.

Table 2 lists the results obtained with CBT and those obtained with W FDA.

Table 1 Bending moments $(\mathrm{kNm})$ for a number of grid points: classical beam theory $(\mathrm{CBT})$ and present study (W- $\Phi$ FDA and M-W FDA)

\begin{tabular}{lccccccc}
\hline Position & CBT & \multicolumn{2}{c}{ Present study } & \multicolumn{2}{c}{ Present study } & \multicolumn{2}{c}{ Present study } \\
X(m) & (exact results) & \multicolumn{2}{c}{ 5-point grid } & \multicolumn{2}{c}{ 9-point grid } & \multicolumn{2}{c}{ 13-point grid } \\
\hline & & W- $\boldsymbol{\Phi}$ & M-W & W- $\boldsymbol{\Phi}$ & M-W & W- $\boldsymbol{\Phi}$ & M-W \\
\hline 0.0 & -74.42 & -50.07 & -67.80 & -65.89 & -72.73 & -70.33 & -73.66 \\
2.0 & 4.19 & -0.63 & 9.15 & 2.47 & 5.45 & 3.36 & 4.75 \\
4.0 & 42.79 & 24.20 & 46.10 & 36.24 & 43.64 & 39.64 & 43.17 \\
6.0 & 41.40 & 24.41 & 43.05 & 35.42 & 41.82 & 38.52 & 41.58 \\
8.0 & 0.00 & 0.00 & 0.00 & 0.00 & 0.00 & 0.00 & 0.00 \\
\hline
\end{tabular}

As Table 1 shows, the results of both approximations converge towards the exact results; however, M-W FDA delivers better results than $\mathrm{W}-\Phi$ FDA for a given grid. The accuracy increases with an increasing number of grid points. M-W FDA yields better results of bending moments than W- $\Phi$ FDA, since results are obtained here through a one-step approximation, whereas results by $\mathrm{W}-\Phi$ FDA are obtained through a two-step approximation ( $\mathrm{w}$ and $\varphi$ are determined in a first step, and the bending moment $\mathrm{M}$ is calculated in a second step). 
TIMOSHENKO BEAM THEORY USING THE FINITE DIFFERENCE METHOD

Table 2 Bending moments ( $\mathrm{kNm}$ ) for a number of grid points: CBT and present study (W FDA)

\begin{tabular}{|c|c|c|c|c|}
\hline $\begin{array}{l}\text { Position } \\
\text { X(m) }\end{array}$ & $\begin{array}{c}\text { CBT } \\
\text { (exact results) }\end{array}$ & $\begin{array}{l}\text { Present study } \\
\text { 5-point grid }\end{array}$ & $\begin{array}{l}\text { Present study } \\
\text { 3-point grid }\end{array}$ & $\begin{array}{r}\text { Present study } \\
\text { 2-point grid }\end{array}$ \\
\hline & & $\mathbf{W}$ & $\mathbf{W}$ & $\mathbf{W}$ \\
\hline 0.0 & -74.42 & -74.42 & -74.42 & -74.42 \\
\hline 2.0 & 4.19 & 4.19 & & \\
\hline 4.0 & 42.79 & 42.79 & 42.79 & \\
\hline 6.0 & 41.40 & 41.40 & & \\
\hline 8.0 & 0.00 & 0.00 & 0.00 & 0.00 \\
\hline
\end{tabular}

The results obtained with W FDA are exact for a uniformly distributed load regardless of the grid. An explanation is the fact that the exact solution for the deflection curve is a fourth-order polynomial, which corresponds to the FDM polynomial hypothesis.

\subsubsection{Beam subjected to a concentrated load}

We analyzed a uniform fixed-pinned beam subjected to a concentrated load, as represented in Figure 15.

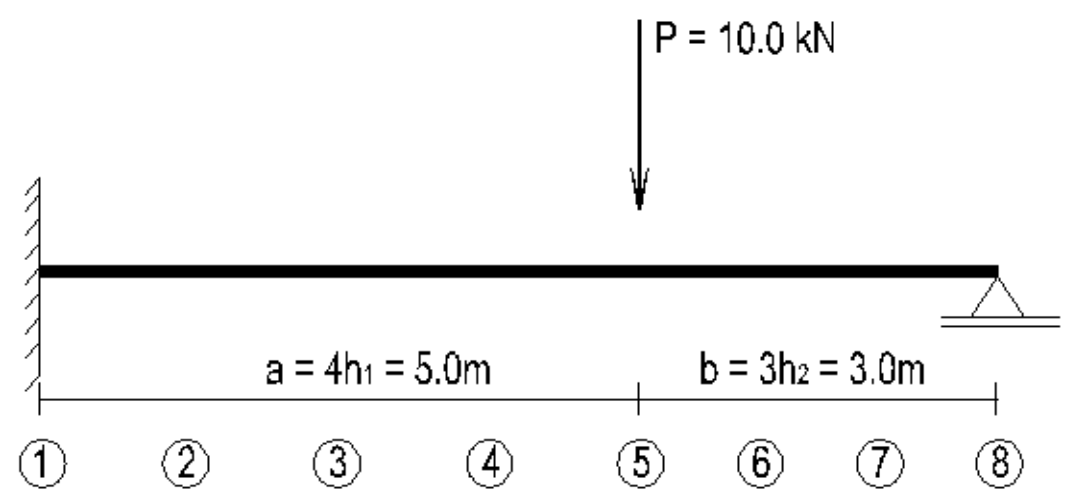

Figure 15 Uniform fixed-pinned beam subjected to a concentrated load

The models showing the grid points (Figure 4a,b and Figure 5a,b) were considered. The beam was calculated using W- $\Phi$ FDA, M-W FDA, and W FDA. Detailed analysis and results are presented in Appendix B and in the Supplementary Material "Fixed-pinned beam subjected to a concentrated load." Table 3 shows the exact results (bending moments) obtained with CBT and those obtained in this study (W- $\Phi$ FDA, M-W FDA, and W FDA). 
Table 3 Bending moments (kNm): CBT, present study (W- $\Phi$ FDA, M-W FDA, and W FDA)

\begin{tabular}{|c|c|c|c|c|c|}
\hline $\begin{array}{l}\text { Position } \\
\mathbf{X}(\mathbf{m})\end{array}$ & $\begin{array}{c}\text { CBT } \\
\text { (exact results) }\end{array}$ & & $\begin{array}{l}\text { t-point grid } \\
\text { m) }+(3 \times 1.0 \mathrm{~m})\end{array}$ & & $\begin{array}{l}\text { Three-point grid } \\
5.0 \mathrm{~m}+\mathbf{3 . 0} \mathrm{m}\end{array}$ \\
\hline & & $\mathbf{W}-\Phi$ & $\mathbf{M}-\mathbf{W}$ & $\mathbf{W}$ & $\mathbf{W}$ \\
\hline$\overline{0.00}$ & -12.16 & -11.96 & -11.80 & -12.16 & -12.16 \\
\hline 1.25 & -5.57 & -5.41 & -5.27 & -5.57 & \\
\hline 2.50 & 1.01 & 1.15 & 1.26 & 1.01 & \\
\hline 3.75 & 7.60 & 7.71 & 7.79 & 7.60 & \\
\hline 5.00 & 14.19 & 14.26 & 14.33 & 14.19 & 14.19 \\
\hline 6.00 & 9.46 & 9.51 & 9.55 & 9.46 & \\
\hline 7.00 & 4.73 & 4.75 & 4.78 & 4.73 & \\
\hline 8.00 & 0.00 & 0.00 & 0.00 & 0.00 & 0.00 \\
\hline
\end{tabular}

The results obtained with $\mathrm{W}-\Phi$ FDA and M-W FDA have gut accuracy. Surprisingly, W- $\Phi$ FDA yields better results than M-W FDA for this load case; this unexpected finding should be investigated in future research. The results obtained using W FDA are exact for a concentrated load regardless of the discretization, since the exact solution for the deflection curve is a third-order polynomial, which is exactly described with the fourth-order polynomial FDM approximation.

\subsubsection{Tapered pinned-fixed beam subjected to a uniformly distributed load}

A tapered pinned-fixed beam subjected to a uniformly distributed load, as shown in Figure 16, was analyzed.

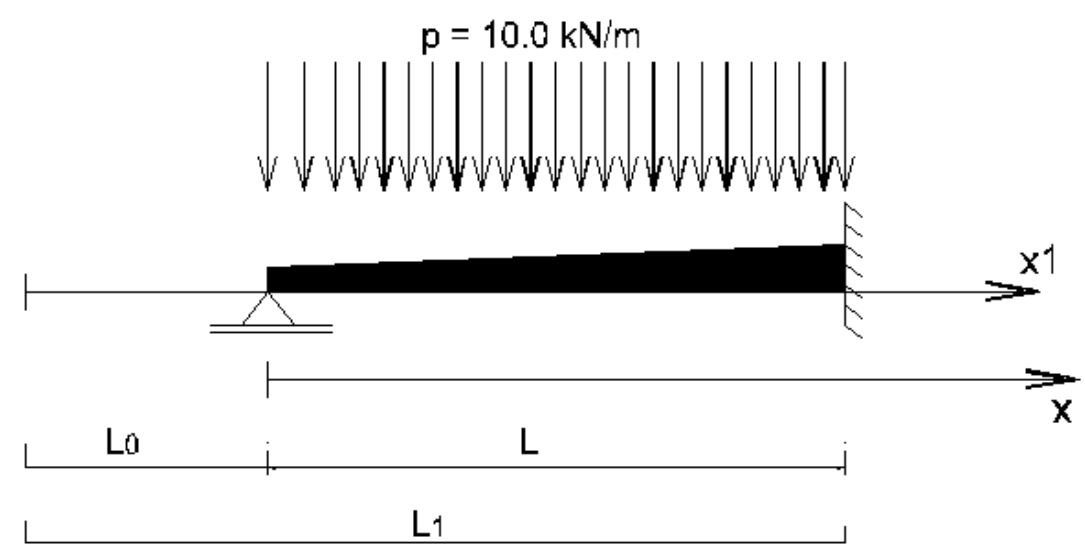

Figure 16 Tapered pinned-fixed beam subjected to a uniformly distributed load 


\section{TIMOSHENKO BEAM THEORY USING THE FINITE DIFFERENCE METHOD}

At position $x_{1}$ of the beam, the second moment of area $\mathrm{I}(x l)$ and the cross-sectional area $\mathrm{A}\left(x_{1}\right)$ are defined as follows:

$$
\begin{aligned}
& I\left(x_{1}\right)=I_{1}\left(x_{1} / L_{1}\right)^{4} \\
& A\left(x_{1}\right)=A_{1}\left(x_{1} / L_{1}\right)^{2}
\end{aligned}
$$

where $\mathrm{I}_{1}$ and $\mathrm{A}_{1}$ are the second moment of area and the cross-sectional area at the fixed end $\mathrm{x}_{1}=\mathrm{L}_{1}$, respectively.

Given $\mathrm{L}=8.0 \mathrm{~m}, \mathrm{~L}_{0}=2.0 \mathrm{~m}$, and $\alpha_{\mathrm{r}}=0.020$.

First, the beam is calculated with the force method of CBT (exact results). Then, the calculation is conducted with FDM using $\mathrm{n}=9,17$, and 25 grid points. Details of the analysis and results are presented in Appendix $\mathrm{C}$ and in the Supplementary Material "Tapered pinned-fixed beam subjected to a uniformly distributed load." Table 4 displays the results obtained with CBT and those obtained in this study (W-Ф FDA and M-W FDA).

Table 4 Bending moments $(\mathrm{kNm})$ for a given number of grid points: CBT and present study (W- $\Phi$ FDA and M-W FDA)

\begin{tabular}{lccccccc}
\hline Position & CBT & \multicolumn{2}{c}{ Present study } & \multicolumn{2}{c}{ Present study } & \multicolumn{2}{c}{ Present study } \\
$\mathbf{X}(\mathbf{m})$ & (exact results) & \multicolumn{2}{c}{ 9-point grid } & \multicolumn{2}{c}{ 17-point grid } & \multicolumn{2}{c}{ 25-point grid } \\
\hline & & $\mathbf{W}-\boldsymbol{\Phi}$ & $\mathbf{M}-\mathbf{W}$ & $\mathbf{W}-\mathbf{\Phi}$ & $\mathbf{M}-\mathbf{W}$ & $\mathbf{W}-\boldsymbol{\Phi}$ & $\mathbf{M}-\mathbf{W}$ \\
\hline 0.00 & 0.00 & 0.00 & 0.00 & 0.00 & 0.00 & 0.00 & 0.00 \\
1.00 & 13.77 & 6.88 & 14.00 & 12.34 & 13.80 & 13.52 & 13.78 \\
2.00 & 17.53 & 21.76 & 18.00 & 20.90 & 17.61 & 19.54 & 17.56 \\
3.00 & 11.30 & 23.55 & 12.01 & 16.90 & 11.41 & 13.84 & 11.34 \\
4.00 & -4.93 & 12.95 & -3.99 & -0.69 & -4.79 & -3.50 & -4.88 \\
5.00 & -31.17 & -16.51 & -29.99 & -30.94 & -30.99 & -31.93 & -31.10 \\
6.00 & -67.40 & -64.15 & -65.99 & -72.91 & -67.18 & -71.03 & -67.32 \\
7.00 & -113.64 & -127.95 & -111.99 & -125.90 & -113.38 & -120.51 & -113.54 \\
8.00 & -169.87 & -205.89 & -167.98 & -189.48 & -169.58 & -180.21 & -169.76 \\
\hline
\end{tabular}

The results of both approximations converge towards the exact results; however, M-W FDA delivers better results than $\mathrm{W}-\Phi$ FDA for a given grid. The accuracy increases with an increasing number of grid points. M-W FDA yields better results of bending moments than $\mathrm{W}-\Phi$ FDA, since the results are obtained here through a one-step approximation, whereas results by the $\mathrm{W}-\Phi$ FDA are obtained through a two-step approximation ( $\mathrm{w}$ and $\varphi$ are determined in the first step, and the moment $\mathrm{M}$ is calculated in the second step). 


\subsection{Second-order analysis}

\subsubsection{Beam subjected to a uniformly distributed load and a compressive force}

A uniform fixed-pinned beam subjected to a uniformly distributed load and a compressive force, as shown in Figure 17, was analyzed.

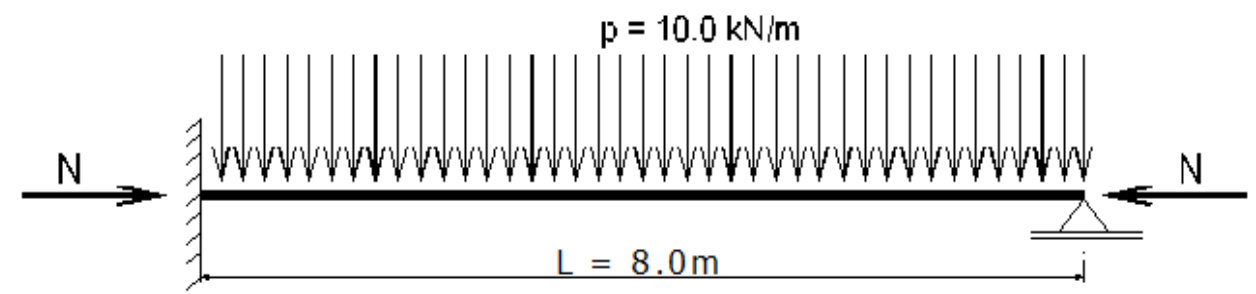

Figure 17 Fixed-pinned beam subjected to a uniformly distributed load and a compressive force

Given $\quad N l^{2} / E I=-3.00, \quad \mathrm{p}=10.0 \mathrm{kN} / \mathrm{m}, \quad 1=8.0 \mathrm{~m} \quad \alpha=\mathrm{EI} / \mathrm{\kappa GAl} \mathrm{l}^{2}=0.02$.

Fogang [6] presented a closed-form expression of the bending moment in a fixed-pinned beam subjected to a uniformly distributed load and a compressive force. In the present study, the analysis was carried out with $n=9,17$, and 25 grid points. Details of the analysis and results are presented in the Supplementary Material "Fixed-pinned beam subjected to a uniformly distributed load and compressive force." Table 5 lists the results obtained using [6] and those in this study (W- $\Phi$ FDA, M-W FDA, and W FDA).

Table 5 Bending moments $(\mathrm{kNm})$ in a fixed-pinned beam: Fogang [6], W- $\Phi$ FDA, M-W FDA, and W FDA

\begin{tabular}{|c|c|c|c|c|c|c|c|}
\hline \multirow[t]{2}{*}{$\begin{array}{l}\text { Position } \\
\text { X(m) }\end{array}$} & \multirow[t]{2}{*}{ Fogang [6] } & \multicolumn{3}{|c|}{$\begin{array}{l}\text { Present study } \\
\text { 9-point grid }\end{array}$} & \multicolumn{3}{|c|}{$\begin{array}{l}\text { Present study } \\
\text { 17-point grid }\end{array}$} \\
\hline & & $\mathbf{W}-\Phi$ & $\mathbf{M}-\mathbf{W}$ & $\mathbf{W}$ & $\mathbf{W}-\Phi$ & $\mathbf{M}-\mathbf{W}$ & $\mathbf{W}$ \\
\hline 0.00 & -91.55 & -75.47 & -105.65 & -92.53 & -86.84 & -95.09 & -91.80 \\
\hline 1.00 & -40.30 & -33.52 & -51.98 & -40.81 & -38.29 & -43.24 & -40.43 \\
\hline 2.00 & 2.36 & 1.01 & -6.86 & 2.27 & 1.98 & 0.04 & 2.33 \\
\hline 3.00 & 34.30 & 26.70 & 27.47 & 34.55 & 32.08 & 32.58 & 34.36 \\
\hline 4.00 & 53.95 & 42.47 & 49.29 & 54.42 & 50.58 & 52.77 & 54.06 \\
\hline 5.00 & 60.33 & 47.68 & 57.52 & 60.88 & 56.61 & 59.61 & 60.46 \\
\hline 6.00 & 53.11 & 42.11 & 51.74 & 53.61 & 49.88 & 52.76 & 53.23 \\
\hline 7.00 & 32.66 & 26.00 & 32.24 & 32.97 & 30.71 & 32.55 & 32.74 \\
\hline 8.00 & 0.00 & 0.00 & 0.00 & 0.00 & 0.00 & 0.00 & 0.00 \\
\hline
\end{tabular}


TIMOSHENKO BEAM THEORY USING THE FINITE DIFFERENCE METHOD

\begin{tabular}{cllcl}
\hline Position & Fogang [6] & \multicolumn{3}{c}{$\begin{array}{c}\text { Present study } \\
\text { 25-point grid }\end{array}$} \\
\hline X(m) & & & M-W & W \\
\hline 0.00 & -91.55 & -89.39 & -93.12 & -91.66 \\
1.00 & -40.30 & -39.38 & -41.61 & -40.36 \\
2.00 & 2.36 & 2.19 & 1.32 & 2.35 \\
3.00 & 34.30 & 33.28 & 33.53 & 34.33 \\
4.00 & 53.95 & 52.40 & 53.42 & 54.00 \\
5.00 & 60.33 & 58.62 & 60.01 & 60.39 \\
6.00 & 53.11 & 51.63 & 52.95 & 53.16 \\
7.00 & 32.66 & 31.76 & 32.61 & 32.70 \\
8.00 & 0.00 & 0.00 & 0.00 & 0.00 \\
\hline
\end{tabular}

The results of all of the approximations converge towards the results of Fogang [6], the accuracy increasing with an increasing number of grid points. W FDA yields here the best results.

\subsubsection{Buckling load of a fixed-pinned beam}

The buckling load of a uniform fixed-free beam, as represented in Figure 18, was determined.

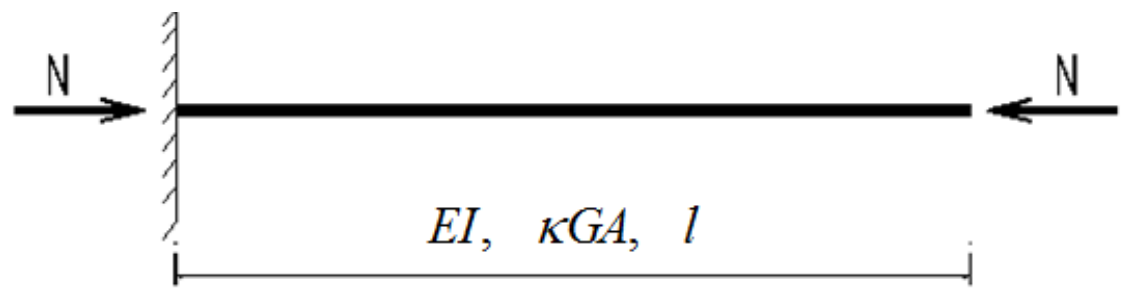

Figure 18 Buckling load of a uniform fixed-free beam

The analysis was conducted with $\mathrm{n}=9$ and 17 grid points. The buckling load $\mathrm{N}_{\mathrm{cr}}$ is defined as follows:

$$
N_{c r}=-\pi^{2} E I /(\beta l)^{2}
$$

Hu et al. [5] presented the following closed-form expression of the buckling load of a uniform fixed-free beam:

$$
N_{c r}=\frac{-\pi^{2} E I}{4 l^{2}\left(1+\pi^{2} \varphi / 48\right)} \quad \varphi=\frac{12 E I}{l^{2} \kappa G A}
$$

The combination of Equations (9c), (66), and (67a) yields the buckling factor $\beta$ as follows:

$$
\beta=\sqrt{4+\pi^{2} \varphi / 12} \equiv \sqrt{4+\pi^{2} \alpha}
$$




\section{TIMOSHENKO BEAM THEORY USING THE FINITE DIFFERENCE METHOD}

Detailed analysis and results are presented in the Supplementary Material "Buckling load of a fixed-free beam." Table 6 shows the results obtained using the formula by $\mathrm{Hu}$ et al. [5] and those obtained in this study (W- $\Phi$ FDA, M-W FDA, and W FDA) for different values of the bending shear factor $\alpha$ (Equation (9c)).

Table 6 Buckling factors $\boldsymbol{\beta}$ of the beam: Hu et al. [5], W-Ф FDA, M-W FDA, and W FDA.

\begin{tabular}{cccccccc}
\hline $\begin{array}{c}\alpha= \\
\text { EI/KGA1 }{ }^{2}\end{array}$ & Hu et al. [5] & \multicolumn{3}{c}{$\begin{array}{c}\text { Present study } \\
\text { 9-point grid }\end{array}$} & & \multicolumn{3}{c}{ Present study } \\
& & & & & 17-point grid & \\
\hline & & W- $\boldsymbol{\Phi}$ & M-W & W & W- $\boldsymbol{\Phi}$ & M-W & W \\
\hline 0.0250 & 2.0608 & 2.1049 & 2.0639 & 2.0711 & 2.0719 & 2.0615 & 2.0634 \\
0.0500 & 2.1198 & 2.1429 & 2.1228 & 2.1301 & 2.1260 & 2.1205 & 2.1224 \\
0.0750 & 2.1772 & 2.1943 & 2.1802 & 2.1875 & 2.1817 & 2.1779 & 2.1798 \\
0.1000 & 2.2332 & 2.2471 & 2.2360 & 2.2425 & 2.2368 & 2.2339 & 2.2357 \\
\hline
\end{tabular}

The results of all of the approximations converge towards those using Hu et al. [5], the accuracy increasing with an increasing number of grid points. M-W FDA yields here the best results.

\subsubsection{Second-order element stiffness matrix of a uniform beam}

The element stiffness matrix of a uniform beam with following characteristics was calculated.

$$
\mathrm{k}=-1.5 \text { (Equation (42c)), } \alpha=0.05 \text { (Equation (9c)), and length } \mathrm{L}=4.0 \mathrm{~m} \text {. }
$$

W FDA and M-W FDA were used with $\mathrm{n}=9,13$, and 17 grid points. $\mathrm{W}-\Phi$ FDA could be considered using formulas developed in Section 2.2.1.1. The stiffness matrix is defined as follows:

$$
K_{T b l}=E I \times\left[\begin{array}{cccc}
T_{T B} & Q_{T B} & -T_{T B} & Q_{T B} \\
& S_{T B} & -Q_{T B} & C_{T B} \\
& & T_{T B} & -Q_{T B} \\
s y m . & & & S_{T B}
\end{array}\right]
$$

Let us now calculate the stiffness matrix of the beam with the following formula presented by Hu et al. [5]:

$$
K_{T b l}=E I \chi \times\left[\begin{array}{cccc}
0 & 0 & (\lambda / L)^{2} & 0 \\
(\lambda / L)^{2} & 0 & 0 & 0 \\
0 & 0 & -(\lambda / L)^{2} & 0 \\
-(\lambda / L)^{2} \cos \lambda & -(\lambda / L)^{2} \sin \lambda & 0 & 0
\end{array}\right] \times\left[\begin{array}{cccc}
1 & 0 & 0 & 1 \\
0 & \chi \lambda / L & 1 & 0 \\
\cos \lambda & \sin \lambda & L & 1 \\
-\chi \lambda / L \sin \lambda & \chi \lambda / L \cos \lambda & 1 & 0
\end{array}\right]^{-1}
$$


The aforementioned characteristics become $\mathrm{P}=1.5 \times \mathrm{EI} / \mathrm{L}^{2}, \chi=1-\mathrm{P} /(\mathrm{ksGA})=1-1.5 \times 0.05=0.925$,

$$
\lambda=\sqrt{P L^{2} / \chi E I}=\sqrt{1.5 / 0.925}=1.273
$$

Details of the analysis and results are presented in Appendix D and in the Supplementary Material "Second-order element stiffness matrix of a uniform beam." Table 7 lists the results obtained using Hu et al. [5] and those obtained in this study.

Table 7 Second-order element stiffness matrix: Hu et al. [5], M-W FDA, and W FDA

\begin{tabular}{cccccccc}
\hline & $\begin{array}{c}\text { Hu et al. } \\
{[5]}\end{array}$ & \multicolumn{2}{c}{ Present study } & \multicolumn{2}{c}{ Present study } & \multicolumn{2}{c}{ Present study } \\
& \multicolumn{2}{c}{ [5-point grid } & \multicolumn{2}{c}{ 13-point grid } & \multicolumn{2}{c}{ 17-point grid } \\
\hline & & M-W & W & M-W & W & M-W & W \\
\hline $\mathrm{T}_{\mathrm{TB}}$ & 0.0917 & 0.0897 & 0.0913 & 0.0908 & 0.0915 & 0.0912 & 0.0916 \\
$\mathrm{Q}_{\mathrm{TB}}$ & 0.2303 & 0.2263 & 0.2295 & 0.2285 & 0.2300 & 0.2293 & 0.2301 \\
$\mathrm{~S}_{\mathrm{TB}}$ & 0.6759 & 0.6682 & 0.6767 & 0.6725 & 0.6763 & 0.6740 & 0.6761 \\
$\mathrm{C}_{\mathrm{TB}}$ & 0.2454 & 0.2369 & 0.2465 & 0.2416 & 0.2459 & 0.2432 & 0.2457 \\
\hline
\end{tabular}

The results of both approximations converge towards those using $\mathrm{Hu}$ et al. [5], the accuracy increasing with an increasing number of grid points. W FDA yields here the best results.

\subsubsection{Free vibration analysis of a fixed-free beam}

The natural frequencies of a fixed-free beam were determined, depending on the bending shear factor $\alpha$ and the coefficient of rotary inertia $\mathbf{k}_{\mathbf{R I}}$. The analysis was conducted with $\mathrm{n}=9,17$, and 25 grid points. Details of the analysis and results are listed in Appendix E and in the Supplementary Material "Vibration analysis of a uniform fixed-free beam." The coefficients $\lambda$ of vibration frequency are defined in Equation (52b). The results obtained in this paper (W- $\Phi$ FDA and M-W FDA) are compared to those obtained using Kruszewski [15], and are listed in Table 8.

Table 8 Coefficients $\boldsymbol{\lambda}$ of natural frequencies (first mode) of a fixed-free beam

\begin{tabular}{cccccccc}
\hline \multirow{2}{*}{$\boldsymbol{\alpha} / \mathbf{k} \mathbf{R I}$} & Kruszewski & \multicolumn{2}{c}{ Present study } & \multicolumn{2}{c}{ Present study } & \multicolumn{2}{c}{ Present study } \\
& {$[\mathbf{1 5}]$} & \multicolumn{2}{c}{ 9-point grid } & \multicolumn{2}{c}{ 17-point grid } & \multicolumn{2}{c}{ 25-point grid } \\
\hline & & $\mathbf{W}-\boldsymbol{\Phi}$ & $\mathbf{M}-\mathbf{W}$ & $\mathbf{W}-\boldsymbol{\Phi}$ & $\mathbf{M}-\mathbf{W}$ & $\mathbf{W}-\boldsymbol{\Phi}$ & $\mathbf{M}-\mathbf{W}$ \\
\hline $0.025 / 0.010$ & 3.2662 & 3.4917 & 3.2290 & 3.3245 & 3.2568 & 3.2922 & 3.2620 \\
$0.025 / 0.015$ & 3.2368 & 3.4549 & 3.2005 & 3.2933 & 3.2276 & 3.2621 & 3.2327 \\
$0.050 / 0.010$ & 3.1159 & 3.2226 & 3.0833 & 3.1431 & 3.1077 & 3.1280 & 3.1122 \\
$0.050 / 0.015$ & 3.0927 & 3.1967 & 3.0607 & 3.1192 & 3.0847 & 3.1045 & 3.0891 \\
\hline
\end{tabular}




\section{TIMOSHENKO BEAM THEORY USING THE FINITE DIFFERENCE METHOD}

The results of both approximations converge towards those using Kruszewski [15], the accuracy increasing with an increasing number of grid points. M-W FDA yields better results than $\mathrm{W}-\Phi$ FDA for a given grid.

\subsubsection{Free vibration analysis of beams resting on Winkler foundation and subjected to a compression force}

The dynamic response of beams resting on an elastic Winkler foundation and subjected to an axial load was determined. A pinned-pinned beam and a fixed-pinned beam were analyzed.

Ghannadiasl [14] analytically solved the case of beams with various boundary conditions, resting on an elastic Winkler foundation and subjected to an axial load. The beams have the following characteristics: Poisson's ratio $v=0.25$, Timoshenko shear coefficient $\kappa=2 / 3$, and coefficient of rotary inertia $k_{R I}=0.01$. The bending shear factor $\alpha$ and the parameter of axial force $\mathrm{k}_{\mathrm{N}}$ (Equation (42c)) are determined as follows

$$
\begin{aligned}
& \alpha=\frac{E I}{\kappa G A L^{2}}=\frac{1}{\kappa} \times \frac{E}{G} \times \frac{I}{A L^{2}}=\frac{1}{2 / 3} \times 2 \times(1+0.25) \times 0.01=0.0375 \\
& N_{x}=-0.6 \times \pi^{2} \times \frac{E I}{L^{2}} \quad \rightarrow \mathrm{k}_{\mathrm{N}}=-5.922
\end{aligned}
$$

The definition of the stiffness of the Winkler foundation in Ghannadiasl [14] has an error: in the denominator, it should be $\mathrm{L}^{4}$ instead of $\mathrm{L}^{2}$.

In the present study, the analysis was conducted with $\mathrm{n}=9,17$, and 33 grid points. Detailed results are listed in the Supplementary Materials "Vibration analysis of a pinned-pinned beam with an axial load" and "Vibration analysis of a fixed-pinned beam with an axial load." Table 9 and Table 10 list the results of Ghannadiasl [14] and those obtained in this study (W- $\Phi$ FDA and M-W FDA).

Table 9 Coefficients $\lambda$ of natural frequencies (first mode) of a pinned-pinned Timoshenko beam under axial load:

Ghannadiasl [14], W- $\Phi$ FDA, and M-W FDA.

\begin{tabular}{lccccccc}
\hline kw & $\begin{array}{c}\text { Ghannadiasl } \\
{[\mathbf{1 4}]}\end{array}$ & \multicolumn{2}{c}{$\begin{array}{c}\text { Present study } \\
\text { 9-point grid }\end{array}$} & \multicolumn{2}{c}{$\begin{array}{c}\text { Present study } \\
\text { 17-point grid }\end{array}$} & \multicolumn{2}{c}{$\begin{array}{c}\text { Present study } \\
\text { 33-point grid }\end{array}$} \\
\hline & & $\mathbf{W}-\boldsymbol{\Phi}$ & $\mathbf{M}-\mathbf{W}$ & $\mathbf{W}-\boldsymbol{\Phi}$ & $\mathbf{M}-\mathbf{W}$ & $\mathbf{W}-\boldsymbol{\Phi}$ & $\mathbf{M}-\mathbf{W}$ \\
\hline 0 & 3.46648 & 4.26106 & 3.35291 & 3.68461 & 3.43818 & 3.52250 & 3.45941 \\
$0.2 \times \pi^{4}$ & 5.52398 & 6.05742 & 5.45397 & 5.66425 & 5.50725 & 5.55952 & 5.51958 \\
$0.4 \times \pi^{4}$ & 7.00019 & 7.43094 & 6.94550 & 7.11207 & 6.98642 & 7.02844 & 6.99674 \\
$0.6 \times \pi^{4}$ & 8.21469 & 8.58696 & 8.16850 & 8.31083 & 8.20306 & 8.23893 & 8.21178 \\
$0.8 \times \pi^{4}$ & 9.27091 & 9.60434 & 9.23034 & 9.35672 & 9.26062 & 9.29253 & 9.26835 \\
\hline
\end{tabular}


TIMOSHENKO BEAM THEORY USING THE FINITE DIFFERENCE METHOD

Table 10. Coefficients $\lambda$ of natural frequencies (first mode) of a fixed-pinned Timoshenko beam under axial load: Ghannadiasl [14], W- - , and M-W.

\begin{tabular}{|c|c|c|c|c|c|c|c|}
\hline \multirow[t]{2}{*}{ kw } & \multirow[t]{2}{*}{$\begin{array}{c}\text { Ghannadiasl } \\
{[14]}\end{array}$} & \multicolumn{2}{|c|}{$\begin{array}{l}\text { Present study } \\
\text { 9-point grid }\end{array}$} & \multicolumn{2}{|c|}{$\begin{array}{l}\text { Present study } \\
\text { 17-point grid }\end{array}$} & \multicolumn{2}{|c|}{$\begin{array}{l}\text { Present study } \\
\text { 33-point grid }\end{array}$} \\
\hline & & $\mathbf{W}-\Phi$ & M-W & $\mathbf{W}-\Phi$ & $\mathbf{M}-\mathbf{W}$ & $\mathbf{W}-\Phi$ & $\mathbf{M}-\mathbf{W}$ \\
\hline 0 & 7.32425 & 7.86512 & 7.09499 & 7.46801 & 7.26653 & 7.36077 & 7.30979 \\
\hline $0.2 \times \pi^{4}$ & 8.50792 & 8.98023 & 8.31146 & 8.63265 & 8.45830 & 8.53955 & 8.49549 \\
\hline $0.4 \times \pi^{4}$ & 9.54555 & 9.97105 & 9.37093 & 9.65748 & 9.50137 & 9.57390 & 9.53447 \\
\hline $0.6 \times \pi^{4}$ & 10.4806 & 10.87162 & 10.32186 & 10.58318 & 10.44038 & 10.50656 & 10.47050 \\
\hline $0.8 \times \pi^{4}$ & 11.3384 & 11.70279 & 11.19194 & 11.43386 & 11.30131 & 11.36259 & 11.32913 \\
\hline
\end{tabular}

The results of both approximations converge towards those of Ghannadiasl [14], the accuracy increasing with an increasing number of grid points. $\mathrm{M}-\mathrm{W}$ FDA yields better results than $\mathrm{W}-\Phi$ FDA for a given grid.

\subsubsection{Free vibration analysis of tapered Timoshenko beams}

The natural frequencies (coefficients $\lambda$ ) of tapered Timoshenko beams were determined. Pinned-pinned, fixed-free, and fixed-fixed beams were considered.

The beams have the following characteristics: Poisson's ratio $v=0.30$, Timoshenko shear coefficient $\kappa=5 / 6$, and coefficient of rotary inertia $\mathrm{k}_{\mathrm{RI}}=0.01$. The bending shear factor is therefore calculated as follows:

$$
\alpha=\frac{E I}{\kappa G A L^{2}}=\frac{1}{\kappa} \times \frac{E}{G} \times \frac{I}{A L^{2}}=\frac{1}{5 / 6} \times 2(1+0.30) \times 0.01=0.0312
$$

Soltani [16] presented results obtained with the power series method (PSM) and those obtained by Hibbitt et al. [17] with the finite element method using ABAQUS software. In this study the analysis was conducted with $\mathrm{n}=9,17,25$, 33 , and 41 grid points for different values of the taper ratio $\left(1-h_{r} / h_{l}\right)$ and support conditions: $h_{l}$ and $h_{r}$ are the heights at the left and the right beam's end, respectively. The reference values Ar and Ir are taken at the left beam's end. Detailed results are listed in the Supplementary Material "Vibration analysis of tapered Timoshenko beams." The results of this study are compared to those of Soltani [16] and Hibbitt et al. [17] in Table 11. 


\section{TIMOSHENKO BEAM THEORY USING THE FINITE DIFFERENCE METHOD}

Table 11 Coefficients $\lambda$ of natural frequencies (first mode) of tapered Timoshenko beams: Soltani [16], Hibbitt et al. [17], and Present study (PS).

\begin{tabular}{|c|c|c|c|c|c|c|c|}
\hline Taper & Soltani [16] & Hibbitt [17] & PS & PS & PS & PS & PS \\
\hline ratio & PSM & ABAQUS & 9-pt grid & 17-pt grid & 25-pt grid & 33-pt grid & 41-pt grid \\
\hline \multicolumn{8}{|c|}{ Fixed-free beam } \\
\hline 0.2 & 3.3307 & 3.3770 & 3.4978 & 3.3734 & 3.3498 & 3.3414 & 3.3375 \\
\hline 0.5 & 3.5591 & 3.6890 & 3.6839 & 3.5895 & 3.5725 & 3.5666 & 3.5639 \\
\hline \multicolumn{8}{|c|}{ Pinned-pinned beam } \\
\hline 0.2 & 7.7160 & 7.7370 & 8.2013 & 7.8419 & 7.7723 & 7.7478 & 7.7364 \\
\hline 0.5 & 6.4442 & 6.4740 & 7.0314 & 6.5924 & 6.5100 & 6.4812 & 6.4678 \\
\hline
\end{tabular}

Fixed-fixed beam

\begin{tabular}{llllllll}
\hline 0.2 & 13.2223 & 13.2380 & 13.7840 & 13.3733 & 13.2903 & 13.2607 & 13.2469 \\
0.5 & 11.9235 & 11.9500 & 12.8356 & 12.1781 & 12.0392 & 11.9891 & 11.9657 \\
\hline
\end{tabular}

The results of both approximations converge towards those of Soltani [16] and Hibbitt et al. [17], whereby the accuracy increases with an increasing number of grid points.

\section{Conclusions}

The FDM-based model developed in this paper enabled, with relative easiness, first-order analysis, second-order analysis, and vibration analysis of Timoshenko beams. The results showed that the calculations conducted as described in this paper were accurate, and the accuracy increased with an increasing number of grid points. Especially in first-order analysis of uniform beams, the results were exact for uniformly distributed and concentrated loads regardless of the discretization. First- and second-order element stiffness matrices (tensile or compressive axial force) in local coordinates were determined. In addition, tapered beams were analyzed.

The following aspects were not addressed in this study but could be analyzed with the model in future research:

Analysis of linear structures, such as frames, through the transformation of element stiffness matrices from local coordinates to global coordinates

$\checkmark \quad$ Second-order analysis of frames free to sidesway, the P- $\Delta$ effect being examined

Timoshenko beams resting on Pasternak foundations

Elastically connected multiple-beam system

However, some study limitations should be acknowledged: 
Large deformation theory

Supplementary Materials: The following files were uploaded during submission:

- "Fixed-pinned beam subjected to a uniformly distributed load"

- "Fixed-pinned beam subjected to a concentrated load"

- "Tapered pinned-fixed beam subjected to a uniformly distributed load"

- "Fixed-pinned beam subjected to a uniformly distributed load and compressive force"

- "Buckling load of a fixed-free beam"

- "Second-order element stiffness matrix of a uniform beam"

- "Vibration analysis of a uniform fixed-free beam

- "Vibration analysis of a pinned-pinned beam with an axial load"

- "Vibration analysis of a fixed-pinned beam with an axial load"

- "Vibration analysis of tapered Timoshenko beams"

\section{Author Contributions:}

\section{Funding:}

\section{Acknowledgments:}

Conflicts of Interest: The author declares no conflict of interest.

\section{Appendix A: Uniform fixed-pinned beam subjected to a uniformly distributed load}

A uniform beam (Figure 14) subjected to a uniformly distributed load was analyzed using the force method of classical beam theory (CBT). The fixed-end moment was the redundant effort.

In the associated statically determinate system, $\mathrm{M}_{0}(x)$ and $\mathrm{V}_{0}(x)$ are the bending moment and the shear force, respectively, due to the distributed load, whereas $\mathrm{m}(x)$ and $\mathrm{v}(x)$ are the bending moment and the shear force, respectively, due to the virtual unit moment at the fixed end. $\mathrm{M}_{0}(x), \mathrm{V}_{0}(x), \mathrm{m}(x)$, and $\mathrm{v}(x)$ can be expressed as follows:

$$
\begin{array}{ll}
M_{0}(x)=p x(l-x) / 2=p l^{2} \xi(1-\xi) / 2 & m(x)=1-x / l=1-\xi \\
V_{0}(x)=p(l / 2-x)=p l(1 / 2-\xi) & v(x)=-1 / l
\end{array}
$$


The fixed-end moment $\mathrm{M}_{1}$ is calculated as follows:

$\delta_{10}=\int_{0}^{l} \frac{M_{0}(x) \times m(x)}{E I} d x+\int_{0}^{l} \frac{V_{0}(x) \times v(x)}{\kappa G A} d x \rightarrow E I \delta_{10}=\frac{p l^{3}}{24}+0$

$\delta_{11}=\int_{0}^{l} \frac{m(x) \times m(x)}{E I} d x+\int_{0}^{l} \frac{v(x) \times v(x)}{\kappa G A} d x \rightarrow E I \delta_{11}=\frac{l}{3}+l \frac{E I}{\kappa G A l^{2}}$

$M_{1}=-\frac{\delta_{10}}{\delta_{11}}=-\frac{p l^{2}}{8+24 \frac{E I}{\kappa G A l^{2}}}$

Combining Equations (A1) and (A4) yields the bending moment at any position $x$, as follows:

$$
M(x)=M_{0}(x)+M_{1} \times m(x)
$$

\section{Appendix B: Uniform fixed-pinned beam subjected to a concentrated load}

A uniform fixed-pinned beam subjected to a concentrated load was analyzed using the force method of CBT. The fixedend moment was the redundant effort. The analysis was conducted similarly to Appendix A.

$$
\begin{aligned}
& E I \delta_{10}=\frac{1}{6} P a b(1+b / l)+0 \quad E I \delta_{11}=\frac{l}{3}+l \frac{E I}{\kappa G A l^{2}} \\
& M_{1}=-\frac{\delta_{10}}{\delta_{11}}=-\frac{P a b(1+b / l)}{l\left(2+6 \frac{E I}{\kappa G A l^{2}}\right)}
\end{aligned}
$$

\section{Appendix C: Tapered pinned-fixed beam subjected to a uniformly distributed load}

A tapered beam (Figure 16) subjected to a uniformly distributed load was analyzed using the force method of CBT. The fixed-end moment was the redundant effort.

In the associated statically determinate system, $\mathrm{M}_{0}(x)$ and $\mathrm{m}(x)$ are the bending moments due to the distributed load and the virtual unit moment at the fixed end, respectively. $\mathrm{V}_{0}(x)$ and $\mathrm{v}(x)$ are the shear forces due to the distributed load and the virtual unit moment at the fixed end, respectively. Let us introduce the dimensionless ordinate $\xi=\mathrm{X} / \mathrm{l}$ and $\xi_{0}=\mathrm{L}_{0} / \mathrm{L}_{1} \cdot \mathrm{M}_{0}(x), \mathrm{V}_{0}(x), \mathrm{m}(x), \mathrm{v}(x), \mathrm{I}(x)$, and $\mathrm{A}(x)$ can be expressed as follows:

$$
\begin{array}{ll}
M_{0}(x)=p x(l-x) / 2=p l^{2} \xi(1-\xi) / 2 & m(x)=x / l=\xi \\
V_{0}(x)=p(l / 2-x)=p l(1 / 2-\xi) & v(x)=1 / l \\
I(x)=I_{1}\left(x_{1} / L_{1}\right)^{4}=I_{1}\left[\xi_{0}+\xi\left(1-\xi_{0}\right)\right]^{4} & \\
A(x)=A_{1}\left(x_{1} / L_{1}\right)^{2}=A_{1}\left[\xi_{0}+\xi\left(1-\xi_{0}\right)\right]^{2} &
\end{array}
$$


Applying Equations (A2) and (A3), the fixed-end moment $\mathrm{M}_{1}$ was determined as follows:

$$
\begin{aligned}
& E I_{1} \delta_{10}=\frac{p l^{3}}{2} \times \int_{0}^{1} \frac{\xi^{2}(1-\xi)}{\left[\xi_{0}+\xi\left(1-\xi_{0}\right)\right]^{4}} d \xi+\alpha_{r} p l^{3} \times \int_{0}^{1} \frac{(1 / 2-\xi)}{\left[\xi_{0}+\xi\left(1-\xi_{0}\right)\right]^{2}} d \xi \\
& E I_{1} \delta_{11}=l \times \int_{0}^{1} \frac{\xi^{2}}{\left[\xi_{0}+\xi\left(1-\xi_{0}\right)\right]^{4}} d \xi+\alpha_{r} l \int_{0}^{l} \frac{1}{\left[\xi_{0}+\xi\left(1-\xi_{0}\right)\right]^{2}} d \xi \\
& M_{1}=-\delta_{10} / \delta_{11}
\end{aligned}
$$

Equations (C2) and (C3) were solved numerically. Combining Equations (C1), (C4), and (A5) yields the bending moment at any position $x$.

For the analysis of the tapered beam using FDM, the parameters $\beta_{\mathrm{M}}^{\prime}(\mathrm{x})$ (Equation (14c)) and $\beta_{\mathrm{V}}^{\prime}(\mathrm{x})$ (Equation (14d)) are calculated as follows:

$$
\begin{aligned}
& \beta_{M}(x)=\left[\xi_{0}+\xi\left(1-\xi_{0}\right)\right]^{4} \rightarrow \beta_{M}^{\prime}(x)=h_{k} \frac{d \beta_{M}(x)}{d x}=4 \beta_{l k}\left(1-\xi_{0}\right)\left[\xi_{0}+\xi\left(1-\xi_{0}\right)\right]^{3} \\
& \beta_{V}(x)=\left[\xi_{0}+\xi\left(1-\xi_{0}\right)\right]^{2} \rightarrow \beta_{V}^{\prime}(x)=h_{k} \frac{d \beta_{V}(x)}{d x}=2 \beta_{l k}\left(1-\xi_{0}\right)\left[\xi_{0}+\xi\left(1-\xi_{0}\right)\right]
\end{aligned}
$$

\section{Appendix D: Second-order element stiffness matrix of a uniform beam}

\section{W FDM approximation}

The static compatibility boundary conditions are expressed using Equations (43b-c) as follows:

$$
\begin{aligned}
& T_{i}=-T_{1}=\rightarrow T_{i}-\left(1+k_{N} \alpha_{r}\right) \frac{-W_{-1}+2 W_{0}-2 W_{2}+W_{3}}{2 h_{k}^{3}}+\beta_{l k}^{2} k_{N} \frac{W_{-1}-8 W_{0}+8 W_{2}-W_{3}}{12 h_{k}^{3}}=0 \\
& M_{i}=M_{1} \rightarrow M_{i}+\left(1+k_{N} \alpha_{r}\right) \frac{-W_{-1}+16 W_{0}-30 W_{1}+16 W_{2}-W_{3}}{12 h_{k}^{2}}=0 \\
& T_{k}=T_{n+1}=\rightarrow T_{k}+\left(1+k_{N} \alpha_{r}\right) \frac{-W_{n-1}+2 W_{n}-2 W_{n+2}+W_{n+3}}{2 h_{k}^{3}}-\beta_{l k}^{2} k_{N} \frac{W_{n-1}-8 W_{n}+8 W_{n+2}-W_{n+3}}{12 h_{k}^{3}}=0 \\
& M_{k}=-M_{n+1} \rightarrow M_{k}-\left(1+k_{N} \alpha_{r}\right) \frac{-W_{n-1}+16 W_{n}-30 W_{n+1}+16 W_{n+2}-W_{n+3}}{12 h_{k}^{2}}=0
\end{aligned}
$$


The geometric compatibility boundary conditions are expressed using Equations (43d) as follows:

$W_{1}=E I_{r} w_{i}$

$E I_{r} \varphi_{1}=\frac{\alpha_{r}}{\beta_{l k}^{2}}\left(1+k_{N} \alpha_{r}\right) \frac{-W_{-1}+2 W_{0}-2 W_{2}+W_{3}}{2 h_{k}}+\frac{W_{-1}-8 W_{0}+8 W_{2}-W_{3}}{12 h_{k}}=E I_{r} \varphi_{i}$

$W_{n+1}=E I_{r} w_{k}$

$E I_{r} \varphi_{n+1}=\frac{\alpha_{r}}{\beta_{l k}^{2}}\left(1+k_{N} \alpha_{r}\right) \frac{-W_{n-1}+2 W_{n}-2 W_{n+2}+W_{n+3}}{2 h_{k}}+\frac{W_{n-1}-8 W_{n}+8 W_{n+2}-W_{n+3}}{12 h_{k}}=E I_{r} \varphi_{k}$

\section{M-W FDM approximation}

The static compatibility boundary conditions are expressed using Equations (43g) as follows:

$$
\begin{aligned}
& T_{i}=-T_{1}=\rightarrow T_{i}+\frac{h_{k}^{2} M_{2}-h_{k}^{2} M_{0}}{2 h_{k}^{3}}+\frac{N_{i} h_{k}^{2}}{E I_{r}} \frac{W_{2}-W_{0}}{2 h_{k}^{3}}=0 \\
& M_{i}=M_{1} \rightarrow h_{k}^{2} M_{i}-h_{k}^{2} M_{1}=0 \\
& T_{k}=T_{n+1}=\rightarrow T_{k}-\frac{h_{k}^{2} M_{n+2}-h_{k}^{2} M_{n}}{2 h_{k}^{3}}-\frac{N_{i} h_{k}^{2}}{E I_{r}} \frac{W_{n+2}-W_{n}}{2 h_{k}^{3}}=0 \\
& M_{k}=-M_{n+1} \rightarrow h_{k}^{2} M_{k}+h_{k}^{2} M_{n+1}=0
\end{aligned}
$$

The geometric compatibility boundary conditions are expressed using Equations (13g) as follows:

$$
\begin{aligned}
& w_{1}=w_{i} \rightarrow W_{1}=E I_{r} \times w_{i} \\
& \varphi_{1}=\varphi_{i} \rightarrow E I_{r} \times \varphi_{1}=\frac{W_{2}-W_{0}}{2 h_{k}}-\frac{\alpha_{r}}{\beta_{l k}^{2}} \frac{h_{k}^{2} M_{2}-h_{k}^{2} M_{0}}{2 h_{k}}=E I_{r} \times \varphi_{i} \\
& w_{n+1}=w_{k} \rightarrow W_{n+1}=E I_{r} \times w_{k} \\
& \varphi_{n+1}=\varphi_{k} \rightarrow E I_{r} \times \varphi_{n+1}=\frac{W_{n+2}-W_{n}}{2 h_{k}}-\frac{\alpha_{r}}{\beta_{l k}^{2}} \frac{h_{k}^{2} M_{n+2}-h_{k}^{2} M_{n}}{2 h_{k}}=E I_{r} \times \varphi_{k}
\end{aligned}
$$




\section{TIMOSHENKO BEAM THEORY USING THE FINITE DIFFERENCE METHOD}

\section{Appendix E}

Kruszewski [15] presented the following equation (Equation (14) in [15]) for the determination of natural frequencies of a uniform fixed-free beam:

$$
2-\frac{k_{B}\left(k_{S}^{2}+k_{R I}^{2}\right)}{\sqrt{1-k_{S}{ }^{2} k_{R I}{ }^{2} k_{B}^{2}}} \sin k_{B} \beta \sinh k_{B} \alpha+\left[k_{B}^{2}\left(k_{S}^{2}-k_{R I}^{2}\right)^{2}+2\right] \cos k_{B} \beta \cosh k_{B} \alpha=0
$$

where $\mathrm{k}_{\mathrm{B}}, \mathrm{k}_{\mathrm{S}}, \mathrm{k}_{\mathrm{RI}}, \alpha$, and $\beta$ are defined in [15] in Equations (3a), (3b), (3c), and (5), respectively.

The following equivalences were noted between the parameters considered by Kruszewski [15] and those considered in this study (PS):

$\mathrm{k}_{\mathrm{S}}^{2}=\alpha, \mathrm{k}_{\mathrm{B}}=\lambda, \mathrm{k}_{\mathrm{RI}}^{2}(\mathrm{~K})=\mathrm{k}_{\mathrm{RI}}(\mathrm{PS})$

\section{References}

[1] Fogang, V. Euler-Bernoulli Beam Theory: First-Order Analysis, Second-Order Analysis, Stability, and Vibration Analysis Using the Finite Difference Method. Preprints 2021, 2021020559 (doi: 10.20944/preprints202102.0559.v2).

[2] M. Kindelan, M. Moscoso, P. Gonzalez-Rodriguez. Optimized Finite Difference Formulas for Accurate High Frequency Components. Mathematical Problems in Engineering. Volume 2016, Article ID 7860618, 15 pages. http://dx.doi.org/10.1155/2016/7860618

[3] Onyia, M.E.; Rowland-Lato, E.O. Determination of the critical buckling load of shear deformable unified beam. IJET 2018, Vol 10 No 3. DOI: 10.21817/ijet/2018/v10i3/181003026

[4] Timoshenko, S.P.; Gere, J.M. Theory of Elastic Stability; McGraw-Hill: New York, USA, 1961.

[5] Hu, Z.P.; Pan, W.H.; Tong, J.Z. Exact solutions for buckling and second-order effect of shear deformable Timoshenko beam-columns based on matrix structural analysis. Appl. Sci. 2019, 9(18), 3814. https://doi.org/10.3390/app9183814

[6] Fogang, V. Timoshenko Beam Theory: Exact Solution for First-Order Analysis, Second-Order Analysis, and Stability. Preprints 2020, 2020110457 (doi: 10.20944/preprints202011.0457.v3).

[7] Mwabora, K. O.; Sigey, J. K.; Okelo, J. A.; Giterere, K. A numerical study on transverse vibration of Euler-Bernoulli beam. IJESIT, Volume 8, Issue 3, May 2019

[8] M. Soltani, A. Sistani, B. Asgarian. Free Vibration Analysis of Beams with Variable Flexural Rigidity Resting on one or two Parameter Elastic Foundations using Finite Difference Method. Conference Paper. The 2016 World Congress on The 2016 Structures Congress (Structures16) 


\section{TIMOSHENKO BEAM THEORY USING THE FINITE DIFFERENCE METHOD}

[9] S. Boreyri, P. Mohtat, M. J. Ketabdari, A.Moosavi. Vibration analysis of a tapered beam with exponentially varying thickness resting on Winkler foundation using the differential transform method. International Journal of Physical Research, 2 (1) (2014) 10-15. doi: 10.14419/iipr.v2i1.2152

[10] K. Torabi, H. Afshari, M. Sadeghi, H. Toghian. Exact Closed-Form Solution for Vibration Analysis of Truncated Conical and Tapered Beams Carrying Multiple Concentrated Masses. Journal of Solid Mechanics Vol. 9, No. 4 (2017) pp. 760-782

[11] Fogang, V. An Exact Solution to the Free Vibration Analysis of a Uniform Timoshenko Beam Using an Analytical Approach . Preprints 2021, 2021010501 (doi: 10.20944/preprints202101.0501.v1).

[12] Y. Yesilce, O. Demirdag, S. Catal: Free vibrations of a multi-span Timoshenko beam carrying multiple spring-mass systems. Sadhana Vol. 33, Part 4, August 2008, pp. 385-401.

\section{https://doi.org/10.1007/s12046-008-0026-1.}

[13] J.T. Katsikadelis. A New Direct Time Integration Method for the Equations of Motion in Structural Dynamics. Conference Paper · July 2011. Third Serbian (28th Yu) Congress on Theoretical and Applied Mechanics. https://www.researchgate.net/publication/280154205

[14] A. Ghannadiasl: An analytical solution for free vibration of elastically restrained Timoshenko beam on an arbitrary variable Winkler foundation and under axial load. Latin American Journal of Solids and Structures 12 (2015) 2417-2438. http://dx.doi.org/10.1590/1679-78251504

[15] Kruszewski, E. T: Effect of transverse shear and rotary inertia on the natural frequency of a uniform beam. National advisory committee for aeronautics, Technical note 1909 https://ntrs.nasa.gov/search.jsp?R=19930082587

[16] Soltani, M: Vibration characteristics of axially loaded tapered Timoshenko beams made of functionally graded materials by the power series method. Numerical Methods in Civil Engineering. July 2017.

DOI:10.29252/nmce.2.1.1

[17] Hibbitt, D., Karlsson, B., Sorensen, P. (2011). Simulia ABAQUS 6. 11 Users’ Manual. 